Document downloaded from:

http://hdl.handle.net/10251/101788

This paper must be cited as:

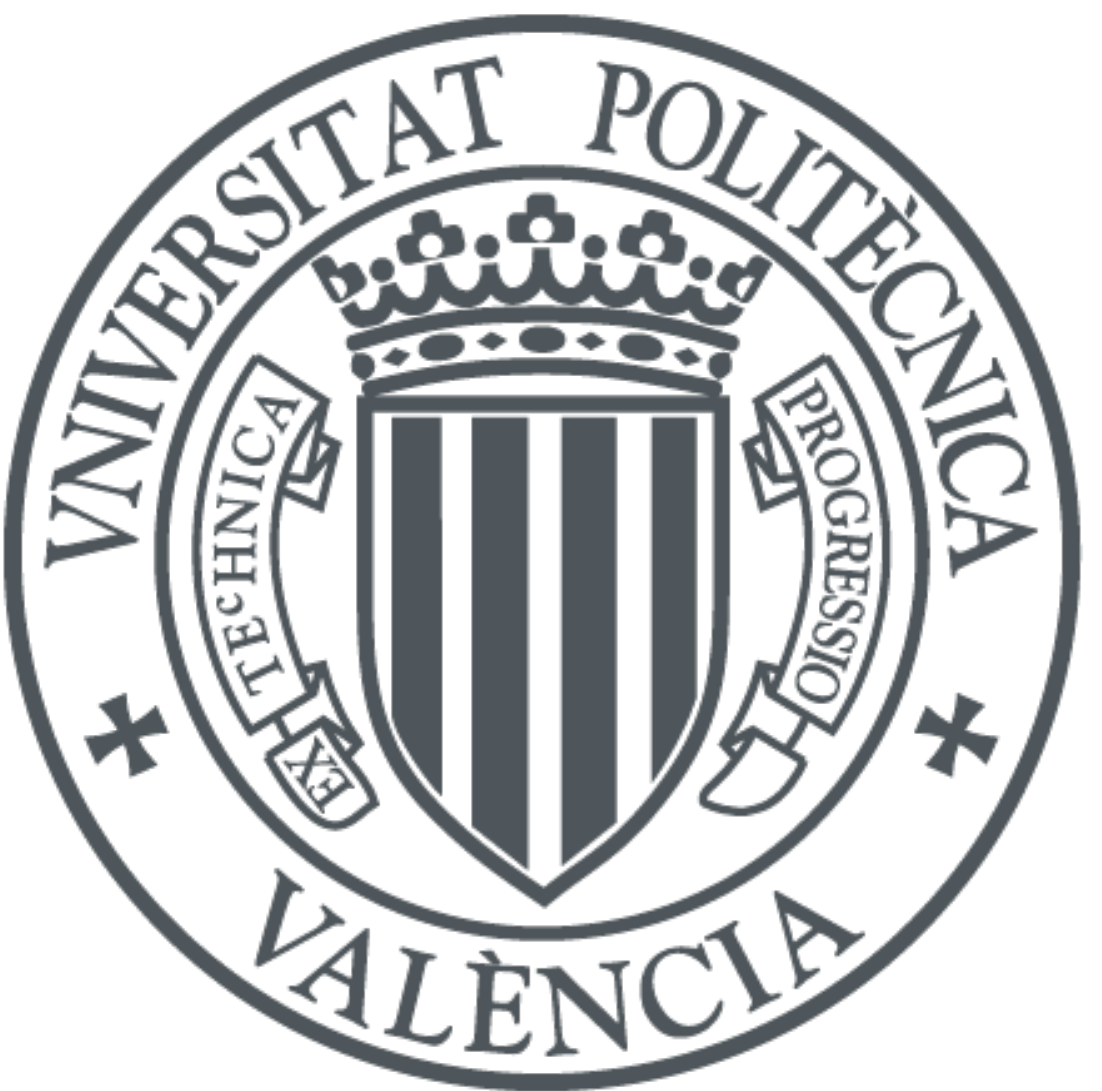

The final publication is available at

https://doi.org/10.1109/TWC.2017.2753784

Copyright Institute of Electrical and Electronics Engineers

Additional Information 


\title{
On the accurate performance evaluation of the LTE-A random access procedure and the access class barring scheme
}

\author{
Israel Leyva-Mayorga, Luis Tello-Oquendo, Vicent Pla, Jorge Martinez-Bauset and Vicente Casares-Giner
}

\begin{abstract}
The performance evaluation of the random access (RA) in LTE-A has recently become a major research topic as these networks are expected to play a major role in future 5G networks. Up to now, the key performance indicators (KPIs) of the RA in LTE-A have been obtained either by performing a large number of simulations or by means of analytic models that, oftentimes, sacrifice precision in exchange of simplicity. In this paper, we present an analytic model for the performance evaluation of the LTE-A RA procedure that incorporates the access class barring (ACB) scheme. By means of this model, each and every one of the key performance indicators suggested by the 3GPP can be obtained with minimal error when compared to results obtained by simulation. To the best of our knowledge, this work presents the most accurate analytic model, which can be easily adapted to incorporate modifications of network parameters and/or extensions to the LTE-A system. In addition, our model of the ACB scheme can be easily incorporated to other analytic models of similar nature without any further modifications.
\end{abstract}

Index Terms-Access class barring (ACB); analytic model; LTE-A; performance evaluation; random access (RA).

\section{INTRODUCTION}

$\mathbf{T}$ HE current LTE-A system has a widely deployed infrastructure, which provides with ubiquitous coverage and global connectivity [1], [2]. As such, LTE-A networks present the best solution for the interconnection of devices (known as user equipments, UEs) and will serve as a foundation for the future development of the IoT and 5G systems [3], [4], [5].

Machine-to-machine communication (M2M) stands for the autonomous exchange of information between UEs; e.g., sensors, actuators, cars, computers or mobile devices inside a common network. In the near future, M2M will enable a myriad of new applications, but presents several design challenges that must be overcome in order to provide an adequate QoS [4], [5], [6], [7]. In M2M applications, usually, a bulk of UEs communicate sparingly with the cellular base stations (known as eNBs in LTE-A) in a highly synchronized manner [5], [8]. While the data packets sent by these UEs are small in size, the large number of access requests may

Manuscript received xxxx yy, zzzz; revised xxxx yy, zzzz. This research has been supported in part by the Ministry of Economy and Competitiveness of Spain under Grants TIN2013-47272-C2-1-R and TEC2015-71932-REDT. The research of I. Leyva-Mayorga was partially funded by grant 383936 CONACYT-Gobierno del Estado de México 2014. The research of L. TelloOquendo was supported in part by Programa de Ayudas de Investigación y Desarrollo (PAID) of the Universitat Politècnica de València.

The authors are with the Instituto ITACA. Universitat Politècnica de València, Camino de Vera s/n. 46022 Valencia, Spain (e-mail: \{isleyma, luiteloq, vpla, jmartinez, vcasares\}@upv.es). exceed the signaling capabilities of the eNBs, which leads to severe network congestion and to the loss of potentially critical information [9], [10].

The UEs access the eNB by means of the random access (RA) procedure; it is performed through the random access channel (RACH) and comprises a four-message handshake: preamble transmission (only allowed in predefined time/frequency resources called random access opportunities, RAOs), random access response (RAR), connection request, and contention resolution messages. The RA procedure of LTE-A, which is described in detail in Section II-B, was devised to handle human-to-human $(\mathrm{H} 2 \mathrm{H})$ traffic. Therefore, it is not efficient at handling the highly-synchronized traffic that is generated by a large number of UEs in M2M applications; hence, severe congestion is likely to occur under these conditions [4], [5], [6], [7], [8]. The frequency and severity of congestion will certainly increase in the coming years due to the rapid increase in the number of interconnected devices (the projected number of mobile-connected devices by 2020 is around 11.6 billion [11]). Hence, efficient access control schemes must be developed; for this, an accurate performance evaluation of the RA procedure must be conducted.

Due to the fact that the RA procedure of LTE-A is hard to model analytically, its performance evaluation is oftentimes conducted by means of simulations [12], [13], [14], [15]. Note that these simulations may be highly time-consuming and the obtained results are not easily reproducible. Hence, an analytic model of the RA procedure is highly valuable.

One of the first efforts to model the RA procedure was presented in [16], but only the first step, preamble transmission, is considered. In fact, there are just a few analytic models for the performance evaluation of the complete RA procedure and their accuracy suffers when compared to simulations [17], [18], [19]. This lack of precision will be clearly observed in Section IV, where we compare the probability distribution of key performance indicators (KPIs) obtained utilizing these models with the ones obtained by simulation. We have observed that the largest error between the existing analytic models and simulations is obtained whenever most of the resources are being utilized (see Section IV for more information on this matter). This fact is a major drawback because the efficient use of resources is of prime importance and one of the main objectives of access control schemes.

Accurately evaluating the access delay of UEs is of utmost importance in time-constrained M2M applications, e.g., health applications [20]. Besides, the access delay of UEs is the most 
neglected KPI in the existing analytic models. For instance, an interesting approach for the performance evaluation of the RA procedure is presented in [21], where the authors employ a technique of fluid approximation. Nevertheless, the described model does not include the limitations of the RAR message and only the average throughput and the average access delay is obtained. Clearly, evaluating the access delay using its average value is not suitable for time-constrained applications; instead, the probability mass function (pmf) of delay should be obtained. A general model for the RACH is presented in [19]; in this study, as in [21], only the average access delay is calculated. The interesting facts that are showcased in [19] are (i) the error of the presented model increases at certain traffic intensities, and (ii) the KPI with the largest relative error is the access delay.

To the best of our knowledge, the most thorough analytic model for the performance evaluation of the RA procedure was presented in [17]. A preliminary version of the latter was presented in [22]. Even though the model presented in [17] was later extended in [18] to incorporate the model of an access control scheme; namely the extended access barring (EAB) scheme, the basic model of the RA procedure is similar. In fact, the model presented in [19] is of similar nature as the one presented in [17]; hence, the obtained error presents a similar behavior in both models. One of the main contributions of [17], [18] is that the probability distribution of access delay can be calculated employing these models, but its accuracy is poor. The main reason for the lack of accuracy of the model presented in [17] is the use of the expected value of the number of preambles decoded by the eNB instead of its pmf; this shortcomming is also described in [23].

In [24], the authors propose a solution to support machinetype communication (MTC) multicast traffic; its benefits are evaluated by means of an analytical model. While the presented model is not as thorough as the one presented, for example in [17], it includes some interesting features. For instance, to the best of our knowledge, it is the only existing model that considers the capacity of the channels that are employed in the RA procedure. The limitations of these channels may impact the access delay of UEs.

Yet another challenge in the analytic modeling of the access of UEs is the modeling of access control schemes, such as the access class barring (ACB). ACB is an access control scheme that redistributes the UE arrivals through time, in such a way that the RACH capacity is not exceeded. During previous studies [25], we have observed that the ACB scheme is capable of effectively reducing the congestion in the RACH if correctly configured.

In [25], we also explained the correct behavior of the ACB scheme as described in [26, Section 5.3.3.11], which had not been correctly evaluated previously (the ACB scheme is described in detail in Section II-A). For instance, the authors of [12] propose a dynamic access control solution and compare its performance with that of a static ACB scheme. Nevertheless, the barring time is assumed to be constant, whereas it is defined in the specification that the barring time must be calculated randomly [26]. This fact negatively affects the performance of the ACB scheme. Also, the limitation in the resources of the RAR message (uplink grants) is not considered.

Analytic models of dynamic ACB schemes (in which the parameters of the ACB scheme are modified throughout the operation of the network) have been presented in [14], [27]. Nevertheless, the behavior of these schemes widely differs from the one defined in the LTE-A specifications [26], [28]. Specifically, the random nature of the barring time and the periodicity of barring update mechanisms are neglected.

In this paper, we present a novel analytic model for the performance evaluation of the access of UEs in LTE-A, where the RA procedure and the ACB scheme are modeled. We describe the process for calculating the following KPI (selected from the ones suggested by the 3GPP [29]):

1) Success probability, defined as the probability to successfully complete the RA procedure within the maximum number of preamble transmissions.

2) Collision probability, defined as the ratio between the total number of preambles transmitted simultaneously by two or more UEs and the total number of available preambles in the period in which accesses occur.

3) Probability distribution of the number of preamble transmissions performed by the UEs that successfully complete the RA procedure.

4) Probability distribution of the access delay.

To the best of our knowledge, this paper presents the first analytic model of the ACB scheme as described in the LTE-A specification [26], [28]. This model can be easily incorporated to other existing analytic models of the RA procedure such as the one presented in [17], [18].

We evaluate the accuracy of our model by comparing the results obtained with both, our model and the one presented in [17] with the ones obtained by simulation. Results show that the error obtained using our analytic model (when compared to the results obtained by simulation) is minimal and surpasses the accuracy of the model presented in [17]. In addition, we have observed that results can be obtained within a few tens of seconds for the selected scenario.

The rest of the paper is organized as follows. We describe and model the RA in LTE-A, i.e., the ACB scheme and the RA procedure in Section II. Then, we describe the process for obtaining each of the KPIs mentioned above in Section III. Section IV presents the results from the performance evaluation of the RA in LTE-A for two cases: no implemented ACB and implemented ACB. Finally, Section V draws our conclusions.

\section{Modeling the ACCESS OF UEs IN LTE-A}

In this section, we describe in detail and model all the steps that must be performed by the UEs to access the eNB.

Before initiating their access attempts, the UEs must acquire the basic configuration of the network, which is broadcast by the eNB through the SystemInformationBlocks. Specifically, the SystemInformationBlockType2 (SIB2) includes, among other parameters, the periodicity of the time/frequency resources in which preamble transmissions are allowed (random access opportunities, RAOs) [4], [10], [26], [30]. That is, the 
Table I

PARAMETERS FOR THE SELECTED RACH CONFIGURATION AND TRAFFIC MODEL 2.

\begin{tabular}{|c|c|}
\hline Parameter & Setting \\
\hline Number of M2M UEs & $n_{\mathrm{M} 2 \mathrm{M}}=30000$ \\
\hline Distribution period & $t_{\text {dist }}=2000 \mathrm{RAOs}$ \\
\hline Pmf of UE arrivals & $p_{A}(i)=\operatorname{Beta}(3,4)$ \\
\hline PRACH Configuration Index & prach-ConfigIndex $\in\{3,6,13\}$ \\
\hline Subframe length & $t_{\mathrm{sf}}=1 \mathrm{~ms}$ \\
\hline Periodicity of RAOs & $t_{\text {rao }} \in\{2,5,10\}$ subframes \\
\hline RAR window size & $w_{\text {rar }}=t_{\text {rao }}$ subframes \\
\hline Available preambles & $n_{\mathrm{r}}=54$ \\
\hline Available uplink grants per subframe & $n_{\mathrm{rar}}=3$ \\
\hline $\begin{array}{l}\text { Available uplink grants per RAR win- } \\
\text { dow }\end{array}$ & $n_{\mathrm{ug}}=w_{\mathrm{rar}} n_{\mathrm{rar}}$ \\
\hline $\begin{array}{l}\text { Maximum number of preamble trans- } \\
\text { missions }\end{array}$ & $k_{\max }=10$ \\
\hline Backoff Indicator & $b_{\mathrm{i}}=20 \mathrm{~ms}$ \\
\hline $\mathrm{ACB}$ barring rate & $p_{\mathrm{acb}}=0.5$ \\
\hline ACB barring time & $t_{\mathrm{acb}}=4$ seconds \\
\hline Preamble processing delay & $d_{\mathrm{p}}=2$ subframes \\
\hline Uplink grant processing delay & $d_{\mathrm{ug}}=5$ subframes \\
\hline$M s g 3$ processing delay & $d_{\mathrm{cr}}=4$ subframes \\
\hline Msg3 RTT & $d_{\mathrm{m} 3}=8$ subframes \\
\hline Msg4 RTT & $d_{\mathrm{m} 4}=5$ subframes \\
\hline $\begin{array}{l}\text { Maximum number } M s g 3 \text { and } M s g 4 \\
\text { transmissions }\end{array}$ & $h_{\max }=5$ \\
\hline $\begin{array}{l}\text { Re-transmission probability for } M s g 3 \\
\text { and } M s g 4\end{array}$ & $p_{\mathcal{E}_{\mathrm{M} 3}}=p_{\mathcal{E}_{\mathrm{M} 4}}=0.1$ \\
\hline $\begin{array}{l}\text { Detection probability for the } k \text { th pream- } \\
\text { ble transmission }\end{array}$ & $p_{\mathcal{D} ; k}=1-1 / e^{k}$ \\
\hline
\end{tabular}

network operates in a slotted channel whose primary time unit is the subframe (of length $t_{\mathrm{sf}}=1 \mathrm{~ms}$ ) and RAOs occur every $t_{\text {rao }}$ subframes; $t_{\text {rao }}$ is determined by the parameter prachConfigIndex.

For the sake of illustration, we use our analytic model to evaluate the performance of the RA in LTE-A under a massive M2M scenario. Throughout this study we follow the recommended $\mathrm{RACH}$ configuration and traffic models described in [29]. Specifically, we select the baseline RACH configuration, prach-ConfigIndex $=6$, and the traffic model 2 with $n_{\mathrm{M} 2 \mathrm{M}}=30000 \mathrm{M} 2 \mathrm{M}$ UEs from [29, Table 6.1.1]. This combination leads to severe network congestion. Other RACH configurations, i.e., prach-ConfigIndex $\in\{3,13\}$, are also considered to further showcase the accuracy of our model; these results are presented in Table II on page 12.

The selected configuration parameters of both the RACH and the traffic model 2 are shown in Table I [29, Table 6.1.1]. The processing delay for each of the steps of the RA procedure is taken from [31, Table 16.2.1-1].

The reason for selecting the baseline RACH configuration, traffic model 2 and $n_{\mathrm{M} 2 \mathrm{M}}=30000$ is that the resulting traffic load (number of UE arrivals per RAO) varies gradually from very low to extremely high and back to very low. Consequently, the utilization of resources ranges from extremely underused to fully utilized (see Section IV). This fact allows us to evaluate the accuracy of our model in each and every one of the traffic loads that can occur in the eNB. Yet another reason for selecting this configuration and traffic model is that most of the studies on the performance evaluation of the RA in LTE-A are performed under these conditions [12], [17], [18], [19].
Hereafter we denote by $i$ and $d$, respectively, the number of elapsed RAOs and number of elapsed subframes. That is, the distributions presented in the following and whose domain is time can be given in either RAOs, $i$, or in subframes, $d$. The distributions that are given in RAOs are used to model the access of the UEs, whereas the distributions that are given in subframes are used to obtain the distribution of access delay. Recall that the duration of a subframe is $1 \mathrm{~ms}$. In the selected $\mathrm{RACH}$ configuration, prach-ConfigIndex $=6$, the periodicity of RAOs is $t_{\text {rao }}=5$ subframes.

Let the random variable (RV) $A$ define the number of RAOs elapsed between the beginning of the distribution period of the M2M UEs and the arrival of a specific UE (the RAO in which the UE schedules the beginning of its RA procedure); the pmf of $A$ for each UE is given by the selected traffic model [29].

Under the traffic model 2, the UE arrivals follow a Beta $(3,4)$ distribution over 10 seconds [29]. As a result, the distribution period of the UE arrivals is $t_{\text {dist }}=2000$ RAOs and the pmf of $A$ is given as

$$
p_{A}(i)=\frac{60 i^{2}\left(t_{\mathrm{dist}}-i\right)^{3}}{t_{\mathrm{dist}}^{6}} .
$$

\section{A. Modeling the ACB scheme}

If the ACB scheme is implemented, all UEs are divided into 16 access classes (ACs). Each UE belongs to one out of the first $10 \mathrm{ACs}$ (from ACs 0 to 9) and can also belong to one or more out of the five special categories (ACs 11 to 15). Thus, M2M devices may be assigned an AC between 0 and 9, and if a higher priority is needed, other classes may be used [28], [32].

The eNB periodically broadcasts, through SystemInformationBlockType2 (SIB2), a mean barring time, $t_{\text {acb }}$ (given in seconds), and a barring rate, $p_{\text {acb }}$, that are applied to all ACs 0-9 and one or more ACs 11-15. Then, upon the beginning of the RA procedure, the UEs that are subject to the ACB scheme determine their barring status with the information provided by the eNB as described in Algorithm 1 [26], [28].

Let $T$ be the RV that defines the number of RAOs that the first preamble transmission of a UE is delayed due to ACB, i.e., the increase in delay due to the ACB scheme. Also, let $X$ be the RV in the domain $x$ that represents the number of barring checks performed by a UE. It is clear that the preamble is transmitted immediately if the UE succeeds in its first barring check $(X=1)$, which occurs with probability $p_{\text {acb. }}$. For the sake of simplicity, we define the function

$$
\delta(i) \equiv \begin{cases}1, & i=0 \\ 0, & \text { otherwise }\end{cases}
$$

then, the pmf of $T$ given $X=1$ is

$$
p_{T \mid X}(i \mid 1)=\delta(i) .
$$

Also, it is clear that the pmf of $T$ given $X=2$ is positive between $i_{T, \min }=\left\lceil 0.7 t_{\mathrm{acb}} / t_{\mathrm{rao}}\right\rceil$ and $i_{T, \max }=\left\lceil 1.3 t_{\mathrm{acb}} / t_{\mathrm{rao}}\right\rceil{ }^{1}$ Its pmf is given as

\footnotetext{
${ }^{1}$ In an abuse of notation, here and in (5) the periodicity of RAOs, given in seconds, is simply denoted as $t_{\text {rao }}$.
} 


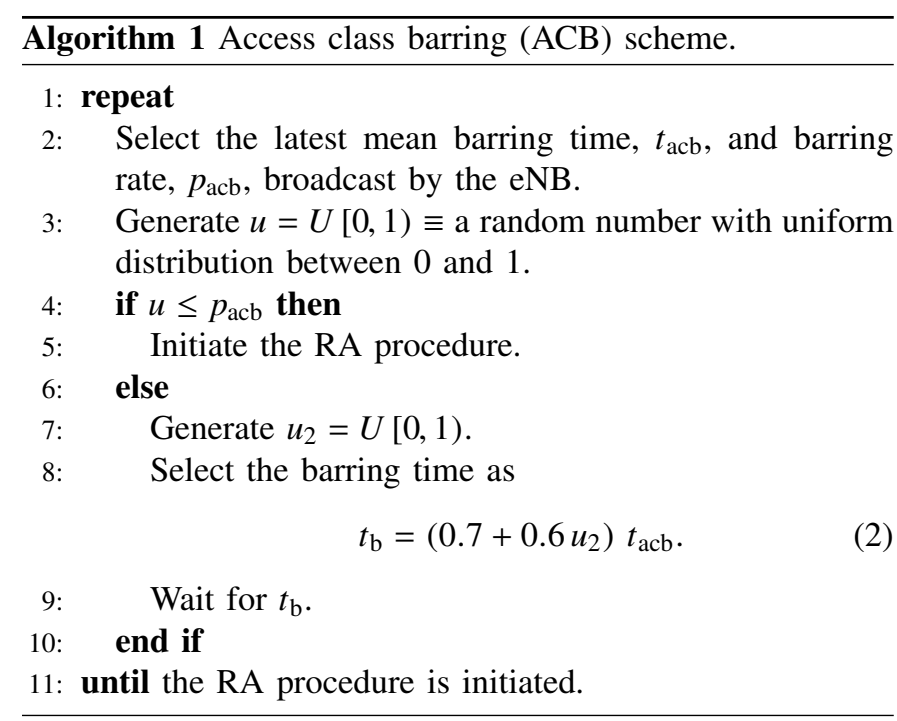

$$
\begin{aligned}
& p_{T \mid X}(i \mid 2) \\
& =\frac{1}{0.6 t_{\mathrm{acb}}} \begin{cases}i t_{\mathrm{rao}}-0.7 t_{\mathrm{acb}}, & i=i_{T, \min } \\
t_{\mathrm{rao}}, & i_{T, \min }<i<i_{T, \max } \\
1.3 t_{\mathrm{acb}}-(i-1) t_{\mathrm{rao}}, & i=i_{T, \max },\end{cases}
\end{aligned}
$$

then, the pmf of $T$ given $X$ can be calculated recursively as

$$
\begin{array}{r}
p_{T \mid X}(i \mid x)=\sum_{\ell=i_{T, \text { min }}}^{i_{T, \text { max }}} p_{T \mid X}(\ell \mid 2) p_{T \mid X}(i-\ell \mid x-1) ; \\
x=3,4, \ldots,
\end{array}
$$

To calculate the pmf of $T$ alone, we first calculate the pmf of $X$ as follows. Each barring check is a single Bernoulli trial as it only has two possible outcomes: success or failure. Hence the pmf of $X$ is given as

$$
p_{X}(x)=p_{\text {acb }}\left(1-p_{\text {acb }}\right)^{x-1}, \quad \text { for } x=1,2, \ldots
$$

which then allows us to calculate the pmf of $T$ as

$$
p_{T}(i)=\sum_{x=1}^{\infty} p_{T \mid X}(i \mid x) p_{X}(x), \quad \text { for } i=0,1,2, \ldots
$$

Note that the sample space of both $T$ and $X$ is infinite; this means that $p_{T}(i) \geq 0, \quad i=0,1,2, \ldots, \infty$. To truncate these sample spaces, let $x_{\max }$ be the maximum number of allowed barring checks; hence the UEs that do not succeed in the first $x_{\max }$ barring checks terminate the ACB scheme and do not perform the RA procedure. Let $p_{\mathcal{E}_{\mathrm{ACB}}}$ be the probability that a UE terminates the ACB scheme, it is given as

$$
p_{\mathcal{E}_{\mathrm{ACB}}}=\left(1-p_{\mathrm{acb}}\right)^{x_{\max }} \text {. }
$$

We calculate $x_{\max }$ as

$$
x_{\max }=\left\lceil\frac{\log p_{\mathcal{E}_{\mathrm{ACB}}}}{\log \left(1-p_{\mathrm{acb}}\right)}\right\rceil,
$$

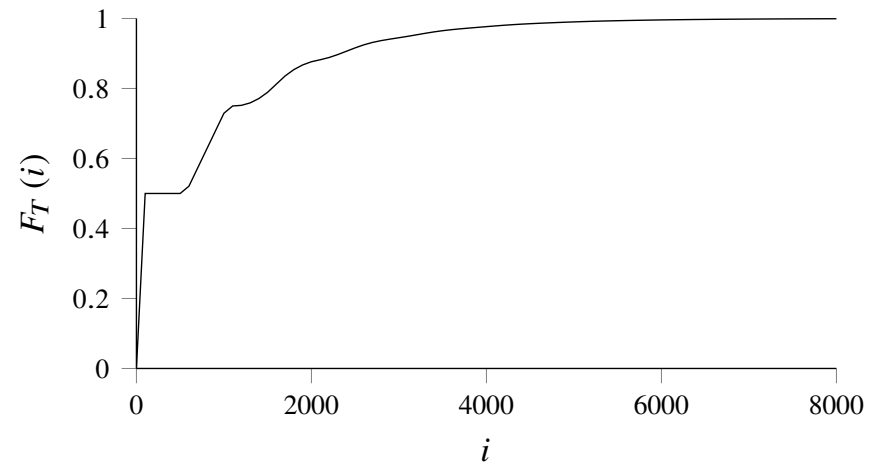

Figure 1. $\mathrm{CDF}$ of the barring time, given $p_{\mathrm{acb}}=0.5, t_{\mathrm{acb}}=4 \mathrm{~s}$ and $p_{\mathcal{E}_{\mathrm{ACB}}}=$ $10^{-5}$.

where $p_{\mathcal{E}_{\mathrm{ACB}}}$ is selected empirically. We approximate $p_{T}(i)$ by truncating (8) as

$$
\begin{aligned}
p_{T^{\prime}}(i) & =p_{T \mid X \leq x_{\max }}(i) \\
& =\frac{1}{1-\left(1-p_{\mathrm{acb}}\right)^{x_{\max }}} \sum_{x=1}^{x_{\max }} p_{T \mid X}(i \mid x) p_{X}(x) .
\end{aligned}
$$

Please observe that $p_{T^{\prime}}(i)$ is indeed a probability distribution and that

$$
p_{T}(i) \approx p_{T^{\prime}}(i), \quad \text { if } p_{\mathcal{E}_{\mathrm{ACB}}} \ll 1 ;
$$

therefore, hereafter we denote $p_{T^{\prime}}(i)$ by simply $p_{T}(i)$. Fig. 1 shows the cumulative distribution function (CDF) of the RV $T$ (in RAOs), $F_{T}(i)$, for the given $p_{\mathrm{acb}}=0.5, t_{\mathrm{acb}}=4 \mathrm{~s}$ and $p_{\mathcal{E}_{\mathrm{ACB}}}=10^{-5}$, hence $x_{\max }=17$.

Let $D_{\mathrm{ACB}}$ be the RV that defines the delay due to the ACB scheme in subframes. It is clear that

$$
\operatorname{Pr}\left\{D_{\mathrm{ACB}}=i t_{\mathrm{rao}}\right\}=\operatorname{Pr}\{T=i\} .
$$

Now that we have obtained the pmf of the UE arrivals, $p_{A}(i)$, and of the barring time, $p_{T}(i)$, we proceed to calculate the number of UEs that begin the RA procedure at each RAO.

Let $n_{\mathrm{m}}(i, k)$ be the number of UEs that are about to perform their $k$ th preamble transmission at the $i$ th RAO. The expected number of UEs that are about to perform their first preamble transmission; i.e., the expected number of UEs that begin the $\mathrm{RA}$ procedure at the $i$ th $\mathrm{RAO}$ is given as

$$
\begin{aligned}
\mathbb{E}\left[n_{\mathrm{m}}(i, 1)\right] & =n_{\mathrm{M} 2 \mathrm{M}} \operatorname{Pr}\{A+T=i\} \\
& =n_{\mathrm{M} 2 \mathrm{M}} \sum_{\ell=0}^{i} p_{A}(\ell) p_{T}(i-\ell) ;
\end{aligned}
$$

the expected number of UEs that are about to perform their $k$ th preamble transmission will later be obtained recursively by means of (33).

Fig. 2 shows the expected number of first preamble transmissions per $\mathrm{RAO}, \mathbb{E}\left[n_{\mathrm{m}}(i, 1)\right]$, for two scenarios. In the first one, no ACB scheme is implemented. This same behavior can be achieved by selecting $p_{\text {acb }}=1$. In the second one, a static ACB scheme is implemented with $p_{\text {acb }}=0.5$ and $t_{\text {acb }}=4$. We will observe in Section IV that this ACB configuration is relevant (for the selected traffic model and $\mathrm{RACH}$ configuration) as it dramatically enhances the network performance. 


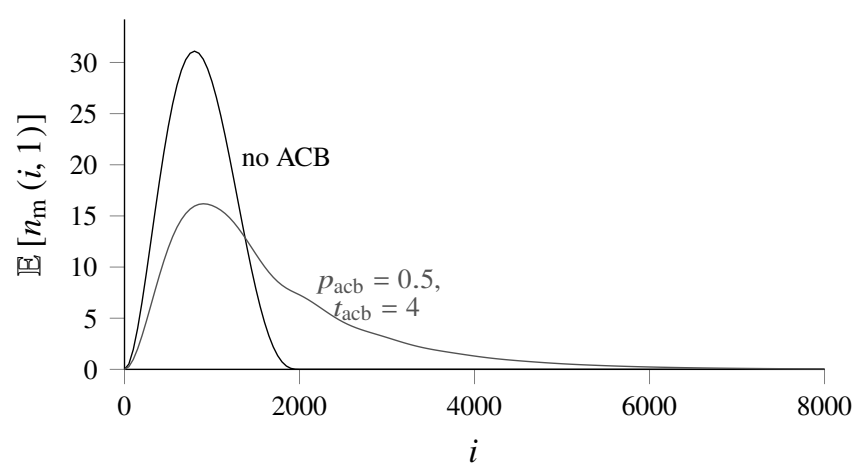

Figure 2. Expected number of first preamble transmissions per RAO, $\mathbb{E}\left[n_{\mathrm{m}}(i, 1)\right]$, under the traffic model 2 for two cases: no ACB scheme, and ACB with $p_{\mathrm{acb}}=0.5, t_{\mathrm{acb}}=4 \mathrm{~s}$.

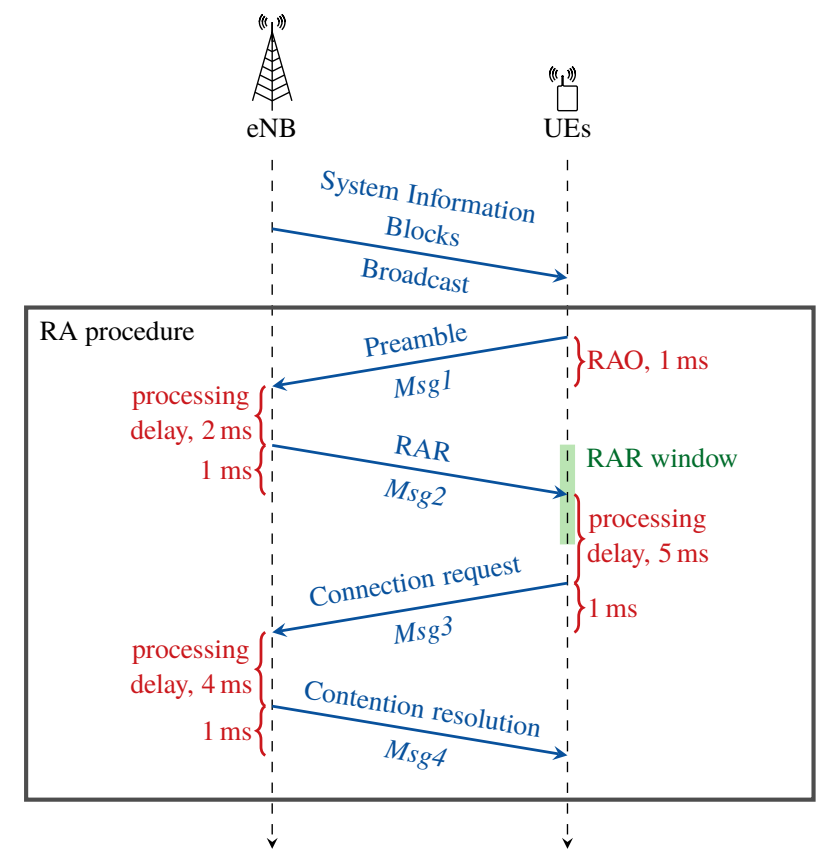

Figure 3. LTE-A contention-based RA procedure.

The UEs that are no more subject to the ACB scheme can now perform the RA procedure as described in the following section.

\section{B. Modeling the RA procedure}

In the following we describe the RA procedure and the process for modeling each of its steps [33], [26], [34], [31]. The four-message handshake of the RA procedure is depicted in Fig. 3.

Preamble (Msg1): At the beginning of the RA procedure, each UE randomly selects one out of the $n_{\mathrm{r}}$ available preambles and sends it to the eNB in a RAO (Msgl). Due to the orthogonality of the different preambles, multiple UEs can access the eNB in the same RAO, using different preambles. If a preamble is transmitted with sufficient power by exactly one UE, it is decoded by the eNB. On the other hand, two outcomes are possible if multiple UEs transmit the same preamble in a RAO:
- The eNB does not decode the transmitted preamble. This may occur if: (i) the eNB determines that the preamble was transmitted by multiple UEs, i.e., based on the received signal power and the time shift between the multiple received copies of the preamble; (ii) the interference caused by the multiple preamble transmissions is too high so that the eNB is not capable of decoding the preamble; or (iii) all the preamble transmissions are lost due to a wireless channel error. Regardless of the cause, the UEs will not receive the RAR message within the RAR window. Then, the implicated UEs will detect the collision.

- The eNB correctly decodes the transmitted preamble. This may occur if: (i) the received power from one of the preamble transmissions is significantly higher than that of the other simultaneous transmissions of the same preamble; i.e., the capture effect, or (ii) all but one of these preamble transmissions are lost due to a wireless channel error. Therefore, the multiple UEs that transmitted the preamble will receive the RAR message and continue with the RA procedure by sending Msg3. The eNB will receive multiple $M s g 3$ s with different data in the same reserved resources and will not transmit Msg4 in response.

The analytical model that is presented in the following has been developed under the assumption that each and every time two or more UEs transmit the same preamble at the same RAO, the eNB does not decode the transmitted preamble. This goes in line with the 3GPP recommendations for the performance evaluation of the RA procedure [29] and with most of the literature [12], [14], [15], [18], [19], [25].

The process to adapt our model so the eNB correctly decodes the preambles transmitted by multiple UEs is not included in this paper due to the lack of space. Nevertheless, we have adapted our model to evaluate the performance of the RA procedure under the assumption that each and every time two or more UEs transmit the same preamble at the same $\mathrm{RAO}$, the eNB correctly decodes the transmitted preamble. In Section IV-C we describe the implications of this assumption; the performance of the RA procedure and the accuracy of our model under this assumption are presented in Table IV on page 13.

In this section, we first obtain the pmfs of the preambles transmitted by exactly one (successful transmissions) and by multiple UEs (collisions) for discrete values of the number of UEs that transmit a preamble at a specific RAO. Then, we derive these same pmfs for any (continuous) value of the expected number of UEs that transmit a preamble at a specific RAO.

The process of preamble selection and transmission can be modeled as a bins and balls problem, as stated in [17]. For this, let $k_{\max }$ be the maximum number of preamble transmissions allowed per UE; this parameter is broadcast by the eNB through the preambleTransMax parameter included in the SIB2 [26]. Also let

$$
n_{\mathrm{m}}(i)=\sum_{k=1}^{k_{\max }} n_{\mathrm{m}}(i, k)
$$


be the total number of balls (UEs that select and transmit a preamble at the $i$ th RAO); each ball is randomly placed in one out of the $n_{\mathrm{r}}$ bins (available preambles). Let $S$ and $C$ be the RVs that represent the number of bins with exactly one ball and the number of bins with more than one ball respectively; namely, the number of preambles transmitted by one (successful) and by multiple UEs (with collision). The domain of $S$ is $s=0,1, \ldots, s_{\max }$, where $s_{\max }=\min \left\{n_{\mathrm{r}}, n_{\mathrm{m}}(i)\right\}$; the domain of $C$ is $c=0,1, \ldots, c_{\max }$, where $c_{\max }=\min \left\{n_{\mathrm{r}}, n_{\mathrm{m}}(i) / 2\right\}$. To solve this problem efficiently, we calculate the joint probability distribution of $S$ and $C$ for a given $n_{\mathrm{m}}(i), p_{S, C}\left(s, c ; n_{\mathrm{m}}(i)\right)$ recursively as

$$
\begin{aligned}
p_{S, C}\left(s, c ; n_{\mathrm{m}}(i)\right)= & \left(\frac{n_{\mathrm{r}}-s+1-c}{n_{\mathrm{r}}}\right) p_{S, C}\left(s-1, c ; n_{\mathrm{m}}(i)-1\right) \\
& +\frac{c}{n_{\mathrm{r}}} p_{S, C}\left(s, c ; n_{\mathrm{m}}(i)-1\right) \\
& +\frac{s+1}{n_{\mathrm{r}}} p_{S, C}\left(s+1, c-1 ; n_{\mathrm{m}}(i)-1\right), \\
& \text { for } s=0,1, \ldots, s_{\max } \text {, and } c=0,1, \ldots c_{\max },
\end{aligned}
$$

given the initial condition $p_{S, C}(0,0 ; 0)=1$.

That is, we derive the probability of having $s$ preambles transmitted by exactly one and $c$ by multiple UEs for a given (discrete) $n_{\mathrm{m}}(i)$ from the case in which $n_{\mathrm{m}}(i)-1$ UEs have already selected their preamble. For this, three possibilities exist:

- $s-1$ preambles are selected by exactly one and $c$ preambles are selected by multiple UEs; then a new UE selects any of the $n_{\mathrm{r}}-(s-1)-c$ preambles that have not been selected by other UEs.

- $s$ preambles are selected by exactly one and $c$ preambles are selected by multiple UEs; then a new UE selects one of the $c$ preambles.

- $s+1$ preambles are selected by exactly one and $c-1$ preambles are selected by multiple UEs; then a new UE selects one of the $s+1$ preambles.

The pmfs of $S$ and $C$ for a given $n_{\mathrm{m}}(i)$ are the marginal probability distributions calculated as

$$
\begin{aligned}
& p_{S}\left(s ; n_{\mathrm{m}}(i)\right)=\sum_{c=0}^{c_{\max }} p_{S, C}\left(s, c ; n_{\mathrm{m}}(i)\right), \\
& p_{C}\left(c ; n_{\mathrm{m}}(i)\right)=\sum_{s=0}^{s_{\max }} p_{S, C}\left(s, c ; n_{\mathrm{m}}(i)\right) .
\end{aligned}
$$

These pmfs can be calculated once for $n_{\mathrm{m}}(i) \in$ $\{1,2, \ldots, v\}\}$, where $v \geq \max \left\{n_{\mathrm{m}}(i)\right\}, \quad i=0,1,2, \ldots$, and stored in a two-dimensional matrix for further use. For the selected scenario, $v \approx 350$.

Fig. $4 \mathrm{a}$ and Fig. $4 \mathrm{~b}$ show the pmf of the number of preambles transmitted by exactly one UE, $p_{S}\left(s ; n_{\mathrm{m}}(i)\right)$, and by multiple UEs, $p_{C}\left(c ; n_{\mathrm{m}}(i)\right)$, for the selected RACH configuration and for characteristic values of $n_{\mathrm{m}}(i)$.

Let $R_{\mathrm{S}}(i)$ and $R_{\mathrm{C}}(i)$ be RVs that define the number of preambles transmitted by exactly one and by multiple UEs at the $i$ th $\mathrm{RAO}$ respectively. We derive the pmfs of $R_{\mathrm{S}}(i)$

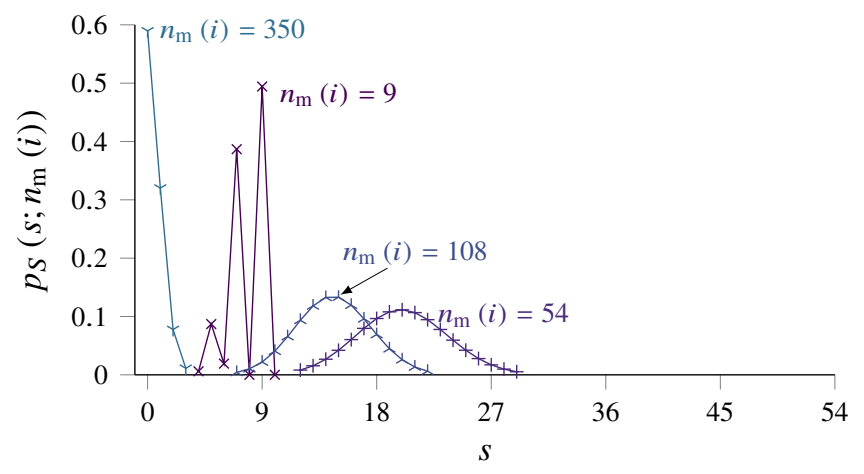

(a)

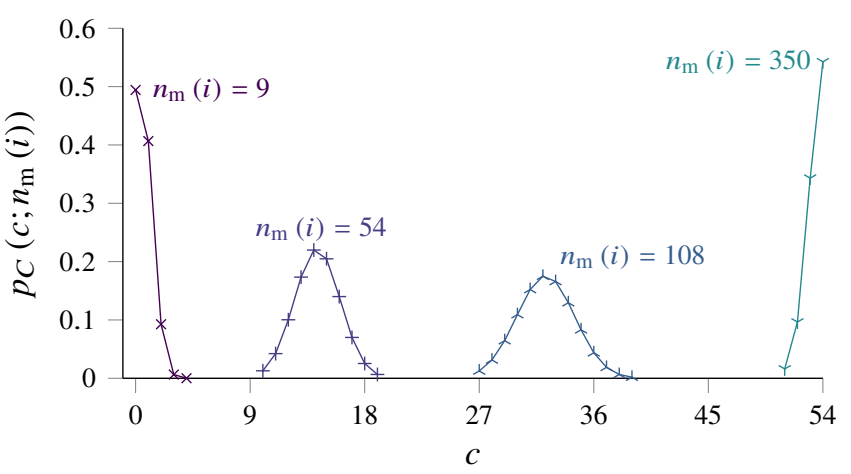

(b)

Figure 4. Probability mass function (pmf) of the number of preambles selected by (a) exactly one UE, $p_{S}\left(s ; n_{\mathrm{m}}(i)\right)$, and (b) by multiple UEs, $p_{C}\left(c ; n_{\mathrm{m}}(i)\right)$, given $n_{\mathrm{r}}=54$ and $n_{\mathrm{m}}(i) \in\{9,54,108,350\}$.

and $R_{\mathrm{C}}(i)$ from the pmfs of $S$ and $C$ by means of the linear interpolation given as

$$
\begin{aligned}
p_{R_{\mathrm{S}}}(s ; i) & \\
= & p_{S}\left(s ;\left\lceil\mathbb{E}\left[n_{\mathrm{m}}(i)\right]\right\rceil\right)\left(\mathbb{E}\left[n_{\mathrm{m}}(i)\right]-\left\lfloor\mathbb{E}\left[n_{\mathrm{m}}(i)\right]\right\rfloor\right) \\
& +p_{S}\left(s ;\left\lfloor\mathbb{E}\left[n_{\mathrm{m}}(i)\right]\right\rfloor\right)\left(1-\mathbb{E}\left[n_{\mathrm{m}}(i)\right]+\left\lfloor\mathbb{E}\left[n_{\mathrm{m}}(i)\right]\right\rfloor\right),
\end{aligned}
$$

$$
\begin{aligned}
p_{R_{\mathrm{C}}}(c ; i) & \\
= & p_{C}\left(c ;\left\lceil\mathbb{E}\left[n_{\mathrm{m}}(i)\right]\right\rceil\right)\left(\mathbb{E}\left[n_{\mathrm{m}}(i)\right]-\left\lfloor\mathbb{E}\left[n_{\mathrm{m}}(i)\right]\right\rfloor\right) \\
& +p_{C}\left(c ;\left\lfloor\mathbb{E}\left[n_{\mathrm{m}}(i)\right]\right\rfloor\right)\left(1-\mathbb{E}\left[n_{\mathrm{m}}(i)\right]+\left\lfloor\mathbb{E}\left[n_{\mathrm{m}}(i)\right]\right\rfloor\right),
\end{aligned}
$$

where

$$
\mathbb{E}\left[n_{\mathrm{m}}(i)\right]=\sum_{k=1}^{k_{\max }} \mathbb{E}\left[n_{\mathrm{m}}(i, k)\right]
$$

is the expected number of UEs that transmit a preamble at each RAO, which is continuous.

Each one of the preambles transmitted by exactly one UE has a certain probability of being correctly decoded at the eNB. We define the event $\mathcal{D}$ as the correct decoding of a preamble transmitted by exactly one UE at a given RAO. The preamble decoding probability for the $k$ th transmitted preamble by a $\mathrm{UE}$ is $p_{\mathcal{D} ; k}$, which depends on the characteristics of the 
wireless channel and the transmission power of the UE (which increases with $k$ due to the power ramping process). The power ramping process and its impact on the preamble detection probability of the $k$ th preamble transmission of each UE was first modeled in [29] as

$$
p_{\mathcal{D} ; k}=1-\frac{1}{e^{k}}
$$

this power ramping model has been adopted in other analytic models such as [17], [18]. From there, we calculate the average preamble detection probability at the $i$ th RAO from (22), which is, by a slight abuse of notation, denoted as

$$
p_{\mathcal{D} ; i}=\frac{1}{\mathbb{E}\left[n_{\mathrm{m}}(i)\right]} \sum_{k=1}^{k_{\max }} p_{\mathcal{D} ; k} \mathbb{E}\left[n_{\mathrm{m}}(i, k)\right],
$$

Next, let $R_{D}(i)$ be the RV that defines the number of preamble transmissions that are correctly decoded by the eNB at the $i$ th $\mathrm{RAO}$; its pmf is calculated as

$$
\begin{array}{r}
p_{R_{\mathrm{D}}}(s ; i)=\sum_{\ell=s}^{n_{\mathrm{r}}}\left(\begin{array}{c}
\ell \\
\ell-s
\end{array}\right)\left(1-p_{\mathcal{D} ; i}\right)^{\ell-s} p_{\mathcal{D} ; i}^{s} p_{R_{\mathrm{S}}}(\ell ; i), \\
\text { for } s=0,1, \ldots, s_{\max } .
\end{array}
$$

Hence, the mean number of decoded preambles at the $i$ th $\mathrm{RAO}$ is given as

$$
\mathbb{E}\left[R_{\mathrm{D}}(i)\right]=\sum_{s=0}^{s_{\max }} s p_{R_{\mathrm{D}}}(s ; i) .
$$

RAR (Msg2): The eNB computes an identifier for each of the successfully decoded preambles and schedules the transmission of a RA response (RAR) message (Msg2). It is transmitted through the physical downlink control channel (PDCCH), along with the Contention resolution message (Msg4) [30]. The RAR message includes, among other data, information about the identification of the detected preamble, time alignment, uplink grants (reserved uplink resources) for the transmission of $M s g 3$, the backoff indicator, and the assignment of a temporary identifier. Exactly two subframes after the preamble transmission has ended (this is the time needed by the eNB to process the received preambles), the UE begins to wait for a time window, RAR window, to receive an uplink grant from the eNB.

There can be up to one RAR message in each subframe, but it may contain multiple uplink grants; each of which is associated to a successfully decoded preamble. The resources of the PDCCH are limited. However, the maximum number of uplink grants per RAR, $n_{\text {rar }}$, can be assumed to be constant because $M s g 2$ (and $M s g 4$ ) are assigned the maximum priority within the PDCCH [29]. The length of the RAR window, $w_{\text {rar }}$ subframes, is broadcast by the eNB through the SIB2 [26]. Hence, there is a maximum number of uplink grants that can be sent within the RAR window. Only the UEs that receive an uplink grant can transmit the $M s g 3$.

Let $M_{\mathrm{U}}(i)$ be the RV that defines the number of UEs that will receive an uplink grant in response to a preamble transmitted in the $i$ th RAO. Let $n_{\mathrm{ug}}=n_{\mathrm{rar}} w_{\text {rar }}$ be the maximum number of uplink grants that can be sent per RAR window, hence, the domain of $M_{\mathrm{U}}(i)$ is $m \in\left\{0,1, \ldots, n_{\mathrm{ug}}\right\}$. The pmf of $M_{\mathrm{U}}(i)$ is given as

$$
p_{M_{\mathrm{U}}}(m ; i)= \begin{cases}p_{R_{\mathrm{D}}}(m ; i), & \text { if } m=0,1, \ldots, n_{\mathrm{ug}}-1 \\ \sum_{s=m}^{n_{\mathrm{r}}} p_{R_{\mathrm{D}}}(s ; i), & \text { if } m=n_{\mathrm{ug}}\end{cases}
$$

and its expected value is

$$
\mathbb{E}\left[M_{\mathrm{U}}(i)\right]=\sum_{m=0}^{n_{\mathrm{ug}}} m p_{M_{\mathrm{U}}}(m ; i) .
$$

Note that $\mathbb{E}\left[M_{\mathrm{U}}(i)\right]$ is indeed the expected number of UEs that successfully complete the first two steps of the RA procedure. Hence the expected number of UEs that successfully complete the first two steps of the RA procedure in their $k$ th preamble transmission can be obtained as

$$
\mathbb{E}\left[M_{\mathrm{U}}(i, k)\right]=\frac{\mathbb{E}\left[M_{\mathrm{U}}(i)\right] \mathbb{E}\left[n_{\mathrm{m}}(i, k)\right] p_{\mathcal{D} ; k}}{\mathbb{E}\left[n_{\mathrm{m}}(i)\right] p_{\mathcal{D} ; i}} .
$$

Then, the expected number of failed UE accesses can be easily calculated as

$$
\mathbb{E}\left[M_{\mathrm{F}}(i, k)\right]=\mathbb{E}\left[n_{\mathrm{m}}(i, k)\right]-\mathbb{E}\left[M_{\mathrm{U}}(i, k)\right] .
$$

Fig. 5 shows the pmf of $p_{R_{\mathrm{S}}}(s ; i), p_{R_{\mathrm{D}}}(s ; i)$ and $p_{M_{\mathrm{U}}}(m ; i)$ for the $i=343$ rd RAO of the selected scenario. At this particular $\mathrm{RAO}, \mathbb{E}\left[n_{\mathrm{m}}(i)\right]=36.05$ and $\mathbb{E}\left[M_{\mathrm{U}}(i)\right]=13.71$.

Backoff: If multiple UEs transmit the same preamble or if the power used for the preamble transmission is not sufficient, then the preamble transmission fails; if the maximum number of preamble transmissions, $k_{\max }$, has not been reached, failed UEs ramp up their power and re-transmit a new randomly chosen preamble in a new RAO. For this, the UE waits for a random time, $U\left(0, b_{\mathrm{i}}\right) \mathrm{ms}$, and then performs a new preamble transmission at the next RAO; $b_{\mathrm{i}}$ is the backoff indicator defined by the eNB, and its value ranges from 0 to $960 \mathrm{~ms}$.

Fig. 6 illustrates the backoff procedure given that multiple transmissions of the same preamble are not decoded by the eNB. It can be seen that the UEs are only aware of a failed preamble transmission if no uplink grant has been received at the end of the RAR window. As a result, the UEs will not be aware of the failed transmission until

$$
d_{\mathrm{f}}=1+d_{p}+w_{\text {rar }}
$$

subframes have elapsed, i.e., one subframe is required for preamble transmission, $d_{p}$ subframes to process the transmitted preambles at the eNB and $w_{\text {rar }}$ is the length of the RAR window.

Each UE keeps track of its number of preamble transmission attempts. When a UE has transmitted $k_{\max }$ preambles without success, the network is declared unavailable by the UE, a RA problem is indicated to upper layers, and the RA procedure is terminated. The value of $k_{\max }$ is defined by the parameter preambleTransMax, broadcast by the eNB through the SIB2 [26].

Let $B$ be the RV that represents the number of RAOs that a UE has to wait due to backoff during its RA procedure. Also, let $K$ be the RV that represents the number of preamble 


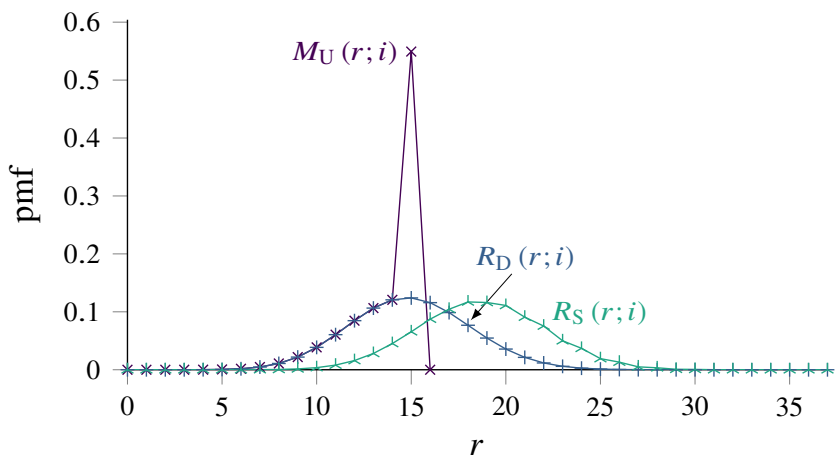

Figure 5. Pmf of the preambles selected by exactly one UE, $p_{R_{\mathrm{S}}}(r ; i)$, preambles decoded at the eNB, $p_{R_{\mathrm{D}}}(r ; i)$, and assigned uplink grants, $p_{M_{\mathrm{U}}}(u ; i)$ for the $i=343 \mathrm{rd} \mathrm{RAO}$.

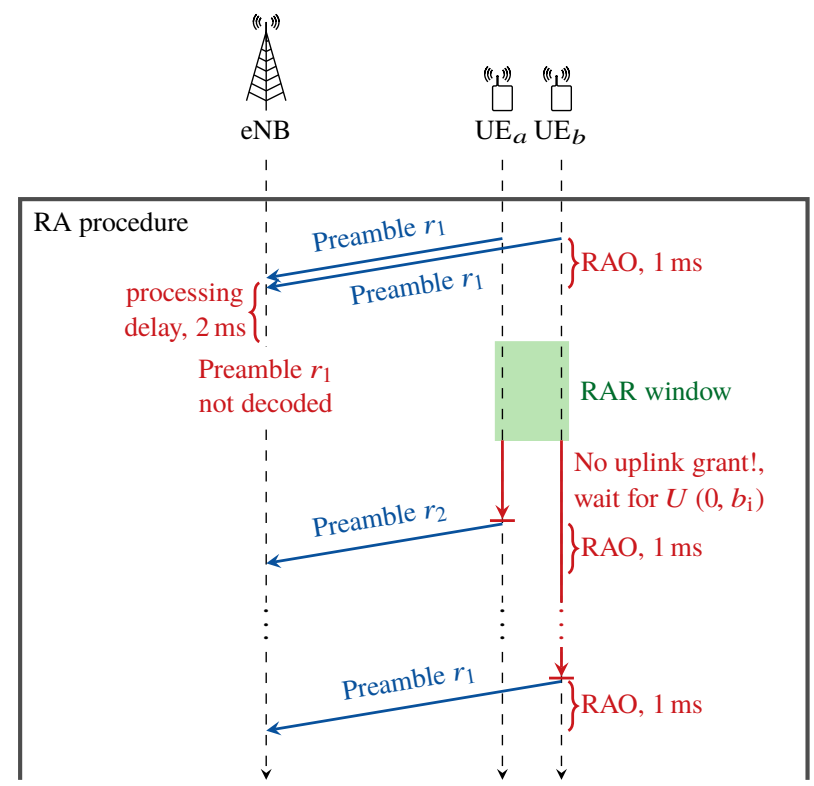

Figure 6. Backoff procedure performed by the failed UEs.

transmissions performed by a UE. If $k=1$, the UE succeeds in its first preamble transmission and backoff is not performed. Therefore, the conditional pmf of $B$ given $k=1$ is given as

$$
p_{B \mid K}(i \mid 1)=\delta(i) .
$$

It is clear that the conditional pmf of $B$ given $k=2$ is positive between $i_{B, \min }=\left\lceil d_{\mathrm{f}} / t_{\mathrm{rao}}\right\rceil$ and $i_{B, \max }=\left\lceil\left(d_{\mathrm{f}}+b_{\mathrm{i}}\right) / t_{\mathrm{rao}}\right\rceil ;^{2}$ and is given as

$$
p_{B \mid K}(i \mid 2)=\frac{1}{b_{\mathrm{i}}} \begin{cases}i t_{\mathrm{rao}}-d_{\mathrm{f}}, & \text { if } i=i_{B, \min } \\ t_{\mathrm{rao}}, & \text { if } i_{B, \min }<i<i_{B, \max } \\ d_{\mathrm{f}}+b_{\mathrm{i}}-(i-1) t_{\mathrm{rao}}, & \text { if } i=i_{B, \max } .\end{cases}
$$

This conditional pmf is of special importance because it allows us to model the backoff process at each RAO by means of the

\footnotetext{
${ }^{2}$ In an abuse of notation, here and in (32) the backoff indicator, given in subframes, is simply denoted as $b_{\mathrm{i}}$.
}

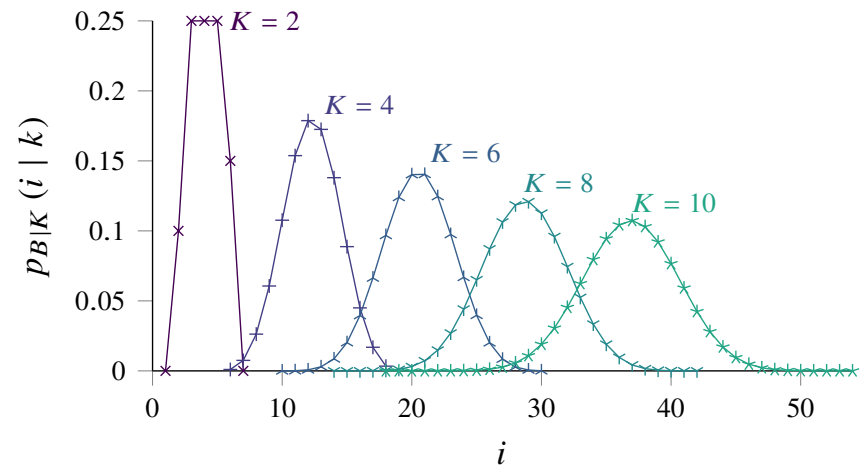

Figure 7. Pmf of the backoff time, $p_{B \mid K}(i \mid k)$, given $K \in\{2,4,6,8,10\}$ for $t_{\text {rao }}=5$ and $b_{\mathrm{i}}=20$.

following recursion

$$
\begin{array}{r}
\mathbb{E}\left[n_{\mathrm{m}}(i, k)\right]=\sum_{j=j_{\min }}^{j_{\max }} \mathbb{E}\left[M_{\mathrm{F}}(i-j, k-1)\right] p_{B \mid K}(j \mid 2), \\
i=1,2, \ldots, i_{\max } ; \quad k=2,3, \ldots, k_{\max } ;
\end{array}
$$

where

$$
i_{\max }=t_{\mathrm{dist}}+\left(k_{\max }-1\right) i_{B, \max }+\left(x_{\max }-1\right) i_{T, \max }
$$

is the last RAO in which a preamble transmission can occur, $j_{\min }=\min \left\{i_{B, \min }, i\right\}, j_{\max }=\min \left\{i_{B, \max }, i\right\}$ and $\mathbb{E}\left[n_{\mathrm{m}}(1, k)\right]=$ 0 for $k \geq 2$.

From (32), the conditional pmf of $B$ given $K$ can be calculated recursively as

$$
\begin{array}{r}
p_{B \mid K}(i \mid k)=\sum_{\ell=i_{B, \min }}^{i_{B, \max }} p_{B \mid K}(\ell \mid 2) p_{B \mid K}(i-\ell \mid k-1), \\
k=3,4, \ldots, k_{\max } .
\end{array}
$$

Fig. 7 shows the pmf of the backoff time (in RAOs) for the selected RACH configuration, in which $t_{\text {rao }}=5$ and $b_{\mathrm{i}}=20$; since $d_{\mathrm{p}}=2$ and $w_{\mathrm{rar}}=5, d_{\mathrm{f}}=8$.

Let $D_{\mathrm{BO}}$ the RV that represents the total number of subframes that a UE has to wait due to backoff during its RA procedure. Clearly, the pmf of $D_{\mathrm{BO}}$ conditioned to the number of preamble transmission attempts, $k$, can be easily calculated as

$$
\operatorname{Pr}\left\{D_{\mathrm{BO}}=i t_{\text {rao }} \mid K\right\}=p_{B \mid K}(i \mid k) .
$$

As with the pmf of the barring time, the pmfs of the backoff time conditioned to the number of preamble transmissions can be obtained once and used repeatedly.

The UEs that have already transmitted $k_{\max }$ preambles without success will terminate the RA procedure. The expected number of UEs that terminate the RA procedure at the $i$ th RAO is simply given as

$$
\mathbb{E}\left[M_{\mathrm{F}}\left(i, k_{\max }\right)\right]=\mathbb{E}\left[n_{\mathrm{m}}\left(i, k_{\max }\right)\right]-\mathbb{E}\left[M_{\mathrm{U}}\left(i, k_{\max }\right)\right] .
$$

Connection request $(\mathrm{Msg3})$ and contention resolution (Msg4): After receiving the corresponding uplink grant, the UE adjusts its uplink transmission time according to the 
received time alignment and transmits a scheduled connectionrequest message, $M s g 3$, to the eNB using the reserved uplink resources; hybrid automatic repeat request (HARQ) is used to protect the message transmission. The RA procedure is concluded when the eNB sends the contention resolution message (Msg4) to the UEs after receiving the connection request; the eNB also applies an HARQ process to protect the transmission of Msg4. If a UE does not receive Msg4 within the Contention Resolution Timer, then it declares a failure in the contention resolution and schedules a new access attempt.

Let $D_{\mathrm{M} 3}$ be the RV that defines the number of subframes elapsed between the first transmission attempt of $M s g 3$ by an $\mathrm{UE}$ and the successful transmission of a $M s g 3$ by the same $\mathrm{UE}$, conditioned to the fact that the transmission of $\mathrm{Msg} 3$ will succeed within the maximum number of attempts. The distribution of $D_{\mathrm{M} 3}, p_{D_{\mathrm{M} 3}}(d)$, depends on the round-trip time, $d_{\mathrm{m} 3}$, the probability of error during the transmission, $p_{\mathcal{E}_{\mathrm{M} 3}}$, and the maximum number of transmission attempts, $h_{\max }$. Let $H$ be the RV that defines the number of attempts that would be required for the successful transmission of Msg3. It is clear that the pmf of $D_{\mathrm{M} 3}$ given $H=h$ is given as

$$
p_{D_{\mathrm{M} 3} \mid H}(d \mid h)=\delta\left(d-(h-1) d_{\mathrm{m} 3}\right) .
$$

Each Msg3 transmission has two possible outcomes: successful or not successful, and the number of transmission attempts is limited to $h_{\max }$. For the sake of simplicity, we consider the UEs that fail the $M s g 3$ (or Msg4) as failed UEs that do not go back to preamble transmission and terminate their RA procedure at this point. This assumption has no impact on the accuracy of our model, since its probability of occurrence,

$$
p_{\mathcal{E}_{\mathrm{M}}}=p_{\mathcal{E}_{\mathrm{M} 3}}^{h_{\max }}+\left(1-p_{\mathcal{E}_{\mathrm{M} 3}}^{h_{\max }}\right) p_{\mathcal{E}_{\mathrm{M} 4}}^{h_{\max }},
$$

is very low for typical values of $p_{\mathcal{E}_{\mathrm{M} 3}}$ and $p_{\mathcal{E}_{\mathrm{M} 4}}$, see Table I on page 3 .

Therefore, the distribution of $D_{\mathrm{M} 3}$ alone can be calculated as

$$
p_{D_{\mathrm{M} 3}}(d)=\frac{1-p_{\mathcal{E}_{\mathrm{M} 3}}}{1-p_{\mathcal{E}_{\mathrm{M} 3}}^{h_{\max }}} \sum_{h=1}^{h_{\max }} p_{\mathcal{E}_{\mathrm{M} 3}}^{h-1} \delta\left(d-(h-1) d_{\mathrm{m} 3}\right)
$$

The distribution of $D_{\mathrm{M} 4}$ can be obtained in the same manner as $D_{\mathrm{M} 3}$ just by substituting the round-trip time, $d_{\mathrm{m} 3}$, with $d_{\mathrm{m} 4}$.

Next, let $D_{\mathrm{M}}$ be the RV that denotes the number of subframes elapsed between the first transmission attempt of $M s g 3$ and the successful transmission of Msg4. The pmf of $D_{\mathrm{M}}$ is given by the sum of $D_{\mathrm{M} 3}$ and $D_{\mathrm{M} 4}$ as

$$
\begin{aligned}
p_{D_{\mathrm{M}}}(d) & =\operatorname{Pr}\left\{D_{\mathrm{M} 3}+D_{\mathrm{M} 4}=d\right\} \\
& =\sum_{\ell=0}^{d} p_{D_{\mathrm{M} 3}}(\ell) p_{D_{\mathrm{M} 4}}(d-\ell)
\end{aligned}
$$

since $D_{\mathrm{M} 3}$ and $D_{\mathrm{M} 4}$ are independent RVs. Fig. 8 shows the CDF of $D_{\mathrm{M} 3}, D_{\mathrm{M} 4}$ and $D_{\mathrm{M}}$ for the selected configuration.

Next, let the RV $M_{\mathrm{S}}(i, k)$ define the number of UEs that successfully transmit their $k$ th preamble at the $i$ th RAO and that will complete the RA procedure a few subframes later. The expected value of $M_{\mathrm{S}}(i, k)$ is given as

$$
\mathbb{E}\left[M_{\mathrm{S}}(i, k)\right]=\left(1-\operatorname{Pr}\left\{\mathcal{E}_{\mathrm{M}}\right\}\right) \mathbb{E}\left[M_{\mathrm{U}}(i, k)\right] .
$$

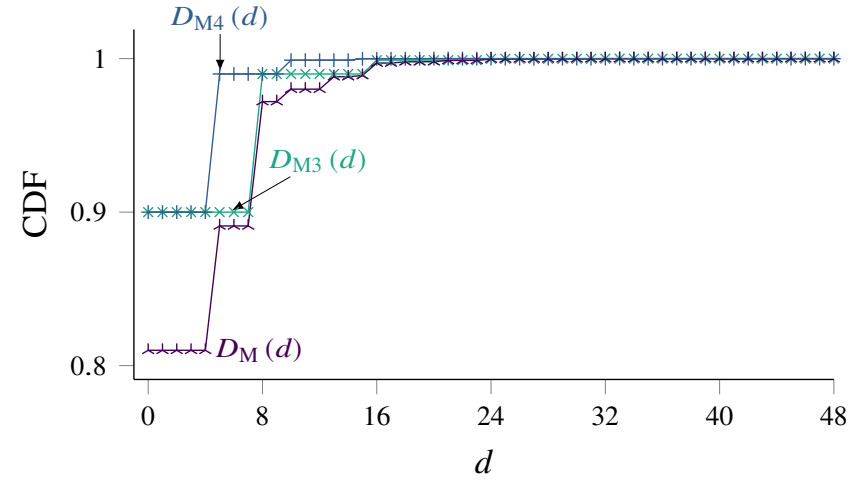

Figure 8. CDF of the access delay due to the transmission of $M s g 3$ and $M s g 4$ for the given probability of error during transmission, $p_{\mathcal{E}_{\mathrm{M} 3}}=p_{\mathcal{E}_{\mathrm{M}}}=0.1$; the RTT of Msg3 is 8 subframes and the the RTT of Msg4 is 5 subframes.

Let $D$ be the RV that defines the number of subframes elapsed since the beginning of the RA until its successful completion. The minimum number of subframes required to successfully complete the RA procedure (minimum access delay) is obtained as

$$
d_{\text {min }}=\min \{d \mid \operatorname{Pr}\{D=d\} \geq 0\}=4+d_{\mathrm{p}}+d_{\mathrm{ug}}+d_{\mathrm{cr}},
$$

since 4 subframes are needed for the transmission of $M s g l$, $M s g 2, M s g 3$, and $M s g 4 ; d_{\mathrm{p}}, d_{\mathrm{ug}}$, and $d_{\mathrm{cr}}$ are the processing delays of the preamble, uplink grant, and connection request messages, respectively [31, Table 16.2.1-1]. Finally, let the RV $D_{\text {min }}$ define the minimum access delay; its pmf is given as

$$
p_{D_{\text {min }}}(d)=\delta\left(d-d_{\min }\right) \text {. }
$$

\section{OBTAINING THE KEY PERFORMANCE INDICATORS}

In this section, we describe in detail the process for obtaining the KPIs for the performance evaluation of the RA in LTE-A. For this, it is necessary to model the RA procedure for each of the RAOs in which a preamble transmission can occur. For the selected scenarios (with and without ACB) this is the period comprised between the beginning of the distribution period, $i=0$, until the last RAO in which a preamble transmission of a UE can be performed, $i_{\max }$.

A UE successfully completes the RA procedure if each and every one of the following conditions are satisfied: (i) it is the only one to select a specific preamble at a RAO; (ii) the UE transmits the preamble to the eNB with sufficient power; (iii) the eNB assigns an uplink grant for the transmitted preamble; (iv) $\mathrm{Msg} 3$ and $\mathrm{Msg} 4$ are transmitted successfully.

Recall that the RV $M_{\mathrm{S}}$ defines the number of successful accesses (the UEs that successfully complete the RA procedure); its expected value for the distribution period is calculated as

$$
\mathbb{E}\left[M_{\mathrm{S}}\right]=\sum_{i=0}^{i_{\max }} \sum_{k=1}^{k_{\max }} \mathbb{E}\left[M_{\mathrm{S}}(i, k)\right] .
$$

That is, $\mathbb{E}\left[M_{\mathrm{S}}\right]$ is the expected number of UEs (out of a total $n_{\mathrm{M} 2 \mathrm{M}} \mathrm{UEs}$ ) that successfully complete the RA procedure. It is obtained by adding the expected number of UEs that successfully complete the RA procedure with any number of preamble transmission for every RAO. 
Let $\mathcal{S}$ be the event defined as the successful completion of the RA procedure. The probability of event $\mathcal{S}$ is the access success probability, given as

$$
p_{\mathcal{S}}=\frac{\mathbb{E}\left[M_{\mathrm{S}}\right]}{n_{\mathrm{M} 2 \mathrm{M}}} .
$$

Let $C$ be the event defined as the transmission of a specific preamble by more than one UE in a RAO (preamble collision). The probability of event $C$ is the collision probability, given as

$$
p_{C}=\frac{1}{\left(i_{\max }+1\right) n_{\mathrm{r}}} \sum_{i=0}^{i_{\max }} \sum_{c=0}^{n_{\mathrm{r}}} c p_{R_{C}}(r ; i) .
$$

Recall that the RV $K$ defines the number of preamble transmissions performed by a UE. As such, the pmf of the number of preamble transmissions performed by the UEs that successfully complete the RA procedure is given as

$$
\begin{array}{r}
p_{K \mid \mathcal{S}}(k)=\operatorname{Pr}\{K=k \mid \mathcal{S}\}=\frac{1}{\mathbb{E}\left[M_{\mathrm{S}}\right]} \sum_{i=0}^{i_{\max }} \mathbb{E}\left[M_{\mathrm{S}}(i, k)\right], \\
\quad \text { for } k=1,2, \ldots, k_{\max },
\end{array}
$$

hereafter simply denoted as $p_{K}(k)$ and its CDF is given as

$$
F_{K}(k)=\operatorname{Pr}\{K \leq k\}, \quad k=1,2, \ldots, k_{\max } ;
$$

the expected value of $K$ can be easily calculated as

$$
\mathbb{E}[K]=\sum_{k=1}^{k_{\max }} k p_{K}(k) .
$$

Let $K_{\phi}$ be the $\phi$ th percentile of the number of preamble transmissions, i.e., the $\phi$ percent of the UEs that successfully complete the RA procedure with $K_{\phi}$ or less preamble transmissions. $K_{\phi}$ is calculated by means of a linear interpolation of $F_{K}(k)$.

Next, we proceed to calculate the access delay. Since the eNB can assign up to $n_{\text {rar }}$ uplink grants per subframe, and the RAR window is comprised of $w_{\text {rar }}$ subframes, the eNB can assign up to $n_{\text {ug }}=w_{\text {rar }} n_{\text {rar }}$ uplink grants per RAO. Let $W$ be the RV in the domain $d \in\left\{0,1, \ldots, w_{\text {rar }}-1\right\}$ that defines the subframe of the RAR window in which the UEs receive the uplink grant. The pmf of $W$ is calculated as

$$
\begin{array}{r}
p_{W}(d)=\frac{1}{\mathbb{E}\left[M_{\mathrm{S}}\right]} \sum_{i=0}^{i_{\max }} \max \left\{0, \min \left\{n_{\mathrm{rar}}, \mathbb{E}\left[M_{\mathrm{S}}(i)\right]-\left(d n_{\mathrm{rar}}\right)\right\}\right\} ; \\
\text { for } d=0,1, \ldots w_{\mathrm{rar}}-1 .
\end{array}
$$

Finally, the pmf of the access delay is given as

$$
\begin{aligned}
p_{D}(d) & =\operatorname{Pr}\{D=d\} \\
& =\operatorname{Pr}\left\{D_{\mathrm{ACB}}+D_{\mathrm{BO}}+D_{\mathrm{M}}+D_{\min }+W=d\right\},
\end{aligned}
$$

i.e., we calculate the pmf of the access delay as the convolution of the pmfs of the barring time, $D_{\mathrm{ACB}}$, the backoff time, $D_{\mathrm{BO}}$, the successful transmission of $M s g 3$ and $M s g 4, D_{\mathrm{M}}$, the minimum access delay, $D_{\min }$, and the subframe in which the uplink grant is received, $W$. These pmfs are calculated in (13), (36), (41), (44), and (51), respectively. The expected value of the access delay, $\mathbb{E}[D]$, and its $\mathrm{CDF}, F_{D}(d)$, are obtained similarly as for $K$ (see (49), (50)). Let $D_{\phi}$ be the $\phi$ th percentile of the access delay, i.e., the $\phi$ percent of the UEs successfully complete the RA procedure with a delay that is less than or equal to $D_{\phi} . D_{\phi}$ is obtained by means of a linear interpolation of $F_{D}(d)$.

\section{Performance evaluation of LTE-A}

In this Section, we showcase the accuracy of our model by presenting the results obtained from the performance evaluation of the RA in LTE-A. The parameters of the selected traffic model and RACH configuration are enlisted in Table I on page 3 .

We have conducted the performance evaluation of the RA in LTE-A in two scenarios: in the first one no ACB scheme is implemented and, in the second one, a static ACB scheme is implemented with $p_{\text {acb }}=0.5$ and $t_{\text {acb }}=4$. During previous studies, we have concluded that the latter configuration efficiently relieves congestion when the UE arrivals occur according to the traffic model 2 [25].

In both scenarios, we compare the accuracy of our analytic model (with respect to simulations) with that of the model presented by C. H. Wei et al. [17]; the latter is hereafter simply denoted as the reference model. Note that while the reference model was the most accurate for the performance evaluation of the RA procedure, it does not incorporate the ACB scheme. To obtain the results derived from the reference model, we have replicated it in each and every aspect as described in [17] and we have incorporated our model of the ACB scheme as described in Section II-A. For this, we have obtained the distribution of UE arrivals after the ACB (as described in Section II-A) and used it as the input to the aforementioned model. Then, we simply perform the convolution between the obtained pmf of access delay and the pmf of the barring time, $p_{D_{\mathrm{ACB}}}(d)$. By doing so, we were able to evaluate the performance of the RA in LTE-A under the static ACB scheme with a model that only incorporates the RA procedure; hereafter, we denote this extension as the extended reference model.

To assess the accuracy of both analytic models, we developed two independent discrete-event simulators. This fact allowed us to confirm our results. The first simulator is C-based and the second one is coded in Octave. In each simulation, $n_{\mathrm{M} 2 \mathrm{M}} \mathrm{UE}$ arrivals were distributed within a period of $t_{\text {dist }}$ RAOs, then the ACB scheme and the contention-based RA procedure were replicated with the parameters listed in Table I. Simulations were run $j$ times until all the cumulative KPIs obtained up to the $j$ th simulation differ from those obtained up to the $(j-1)$ th simulation by less than 0.01 percent. For all of the KPIs presented in Table II and in Table III, the relative margin of error was less than 0.5 percent at a 95 percent confidence level.

\section{A. RA in LTE-A with no ACB scheme}

We begin our analysis by comparing the expected number of successful accesses at each RAO. $\mathbb{E}\left[M_{\mathrm{S}}(i)\right]$ obtained by simulation, by the reference model [17], and by our model. In Fig. 9a we illustrate the comparison whereas in Fig. 9b we 


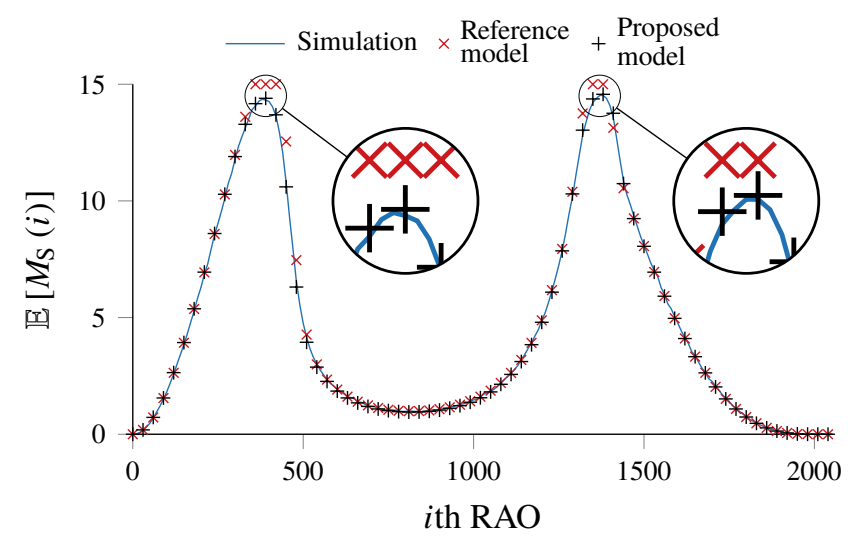

(a)

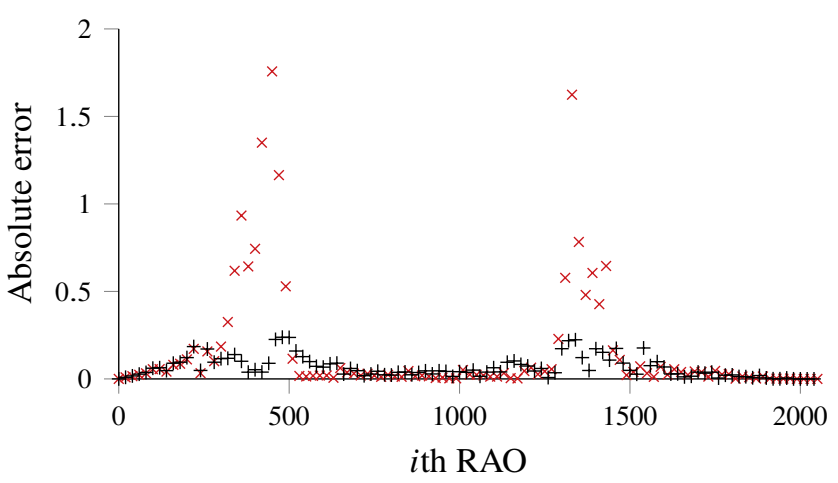

(b)

Figure 9. (a) Comparison and (b) absolute error of the expected number of successful accesses at each RAO, $\mathbb{E}\left[M_{\mathrm{S}}(i)\right]$, obtained by simulation, by the reference model [17] and by our proposed model; no ACB scheme.

show the absolute error of the calculated $\mathbb{E}\left[M_{\mathrm{S}}(i)\right]$ at each RAO. From Fig. 9a and Fig. 9b it can be clearly observed that the results obtained by both models and by simulation are extremely similar for most of the RAOs. The most notorious exception of this is observed for the reference model in the RAOs where $\mathbb{E}\left[M_{\mathrm{S}}(i)\right] \approx 15$, where an absolute error of up to 2 successful accesses per RAO is obtained. The main reason for this is that the number of uplink grants per RAO is calculated from the expected number of decoded preambles. As a result, the reference model overestimates the number of successful accesses for the selected scenario. This problem has also been described in [19].

Note that, by using the pmf of the expected number of decoded preambles, this error is not present in our model (see (26) and Fig. 5), as the absolute error obtained in every RAO is minimal, hence our model is extremely accurate.

To provide with an in-depth look at the accuracy of our model, we show the KPIs obtained by simulation and the relative error between these KPIs and the ones obtained by both of the analytic models in Table II. As shown in Table II, these results were obtained for three different RACH configurations: prach-ConfigIndex $=3,6,13$. Hence, the RAO periodicity is $t_{\text {rao }}=2,5,10$ respectively.

From Table II it can be seen that each one of the KPIs obtained by our analytic model are extremely similar to the ones obtained by simulation. In contrast, the use of the

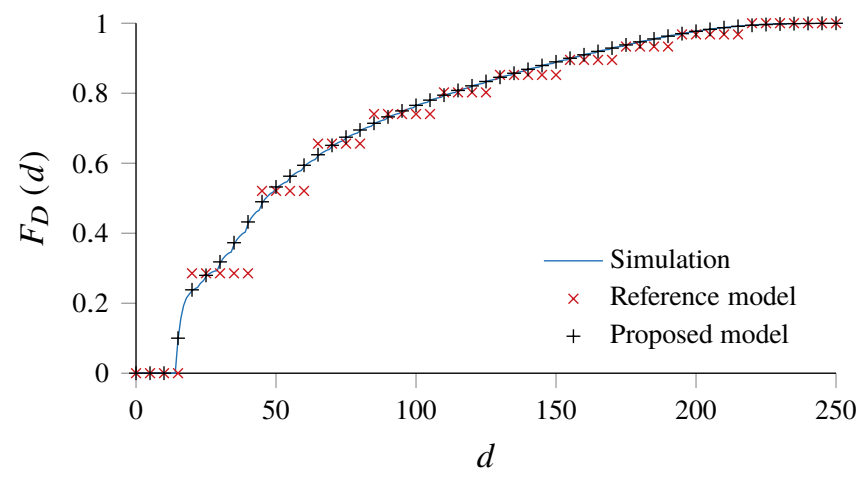

Figure 10. CDF of the access delay, $F_{D}(d)$; no ACB scheme.

reference model leads to an error larger than two percent for several KPIs. Specifically, the use of the reference model oftentimes results in a high error in the access delay. The reason for such a significant error in the latter is, once again, the use of expected values instead of the pmf. Specifically, the expected subframe of the RAR window in which the uplink grant is received and the expected delay due to the transmission of Msg3 and Msg4 are used, not the pmf. The result of this is the step-like function of the CDF of access delay that is depicted in Fig. 10.

\section{B. RA in LTE-A with a static ACB scheme}

Next, we proceed to evaluate the accuracy of our model in an scenario in which a static ACB scheme is implemented. For this, we have selected the following ACB configuration parameters: $p_{\text {acb }}=0.5$ and $t_{\text {acb }}=4$. Table III shows the KPIs obtained in this scenario; as it can be seen, the selected configuration of the static ACB scheme sharply increases the success probability, $p_{\mathcal{S}}$.

As a baseline for this analysis, we show in Fig. 11a the expected number of successful accesses per RAO, $\mathbb{E}\left[M_{\mathrm{S}}(i)\right]$, and in Fig. 11b its absolute error (with respect to simulations) for our analytic model and for that of the extended reference model (which incorporates our ACB model). Again, the error observed for most RAOs is extremely close to zero, except for the extended reference model in those RAOs where $\mathbb{E}\left[M_{\mathrm{S}}(i)\right] \approx 15$. Consequently, a large error is obtained in the success probability, $p_{\mathcal{S}}$, and in the expected number of preamble transmissions, $\mathbb{E}[K]$. This fact can be clearly observed in Table III, where we show the KPIs obtained by simulation and the relative error between these KPIs and the ones obtained by both of the analytic models.

Table III also shows that the error obtained by both analytic models in the 95th percentile of access delay is very low. Nevertheless, a very large error in the 50th percentile of the access delay is obtained by means of the extended reference model. This is a special case, caused by the shape of the CDF of the barring time and the lack of accuracy of the reference model.

In Fig. 12a we show the overall view of the CDF of access delay obtained by simulation and by both analytic models; in Fig. 12b we show in detail these CDFs for the first 250 
Table II

KPIS OBTAINED BY SIMULATION AND THE RELATIVE ERROR OBTAINED BY THE REFERENCE MODEL (RM) AND BY OUR PROPOSED MODEL (PM) FOR DIFFERENT SETTINGS OF prach-ConfigIndex; NO ACB SCHEME.

\begin{tabular}{|c|c|c|c|c|c|c|c|c|c|}
\hline \multirow[b]{2}{*}{ KPI } & \multicolumn{3}{|c|}{$\begin{array}{r}\text { prach-ConfigIndex }=3 \\
\text { Rel. error (\%) }\end{array}$} & \multicolumn{3}{|c|}{$\begin{array}{r}\text { prach-ConfigIndex }=6 \\
\text { Rel. error }(\%)\end{array}$} & \multicolumn{3}{|c|}{$\begin{array}{r}\text { prach-ConfigIndex }=13 \\
\text { Rel. error }(\%)\end{array}$} \\
\hline & Simulation & $\mathrm{RM}$ & PM & Simulation & $\mathrm{RM}$ & PM & Simulation & $\mathrm{RM}$ & PM \\
\hline Success probability & $66.44 \%$ & 0.35 & 0.08 & $31.33 \%$ & 2.70 & 0.29 & $9.89 \%$ & 0.59 & 0.36 \\
\hline Collision probability & $18.02 \%$ & 1.15 & 0.13 & $43.48 \%$ & 1.63 & 0.20 & $60.02 \%$ & 0.02 & 0.09 \\
\hline \multicolumn{10}{|l|}{ Number of preamble transmissions } \\
\hline Expected value & 4.10 & 1.39 & 0.69 & 3.45 & 2.90 & 0.97 & 3.23 & 0.35 & 0.76 \\
\hline 10th percentile & 1.00 & 0.00 & 0.00 & 1.00 & 0.00 & 0.00 & 1.00 & 0.00 & 0.00 \\
\hline 50th percentile & 2.87 & 3.19 & 1.54 & 1.98 & 3.60 & 1.71 & 1.83 & 0.48 & 1.29 \\
\hline 90th percentile & 8.01 & 0.28 & 0.08 & 7.30 & 2.35 & 0.48 & 6.93 & 0.22 & 0.50 \\
\hline 95th percentile & 8.95 & 0.11 & 0.02 & 8.57 & 1.07 & 0.20 & 8.38 & 0.20 & 0.16 \\
\hline \multicolumn{10}{|l|}{ Access delay } \\
\hline 10th percentile & $15.00 \mathrm{~ms}$ & 26.67 & 0.10 & $15.08 \mathrm{~ms}$ & 25.97 & 0.55 & $15.01 \mathrm{~ms}$ & 59.92 & 0.30 \\
\hline 50th percentile & $54.05 \mathrm{~ms}$ & 10.59 & 1.64 & $46.93 \mathrm{~ms}$ & 12.82 & 2.30 & $54.16 \mathrm{~ms}$ & 0.59 & 2.16 \\
\hline 90th percentile & $136.66 \mathrm{~ms}$ & 19.26 & 0.12 & $155.60 \mathrm{~ms}$ & 10.62 & 0.33 & $196.35 \mathrm{~ms}$ & 2.34 & 0.38 \\
\hline 95th percentile & $153.52 \mathrm{~ms}$ & 19.17 & 0.09 & $182.59 \mathrm{~ms}$ & 6.51 & 0.34 & $236.56 \mathrm{~ms}$ & 10.08 & 0.24 \\
\hline
\end{tabular}

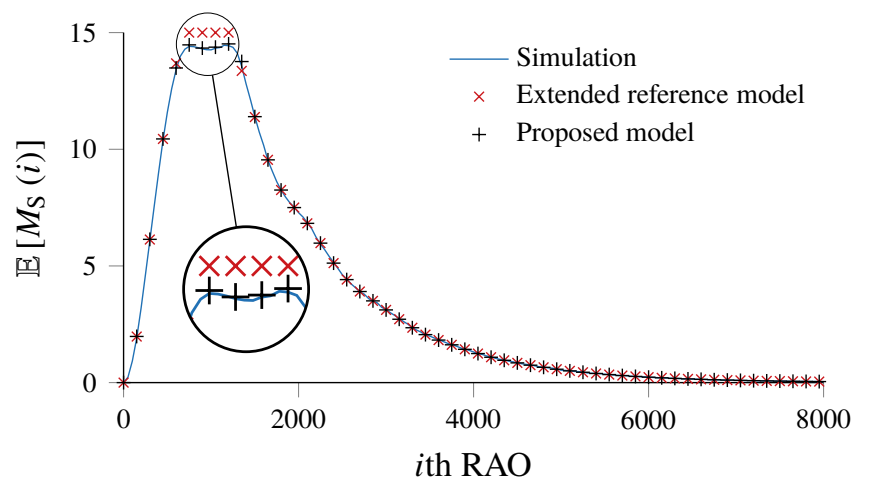

(a)

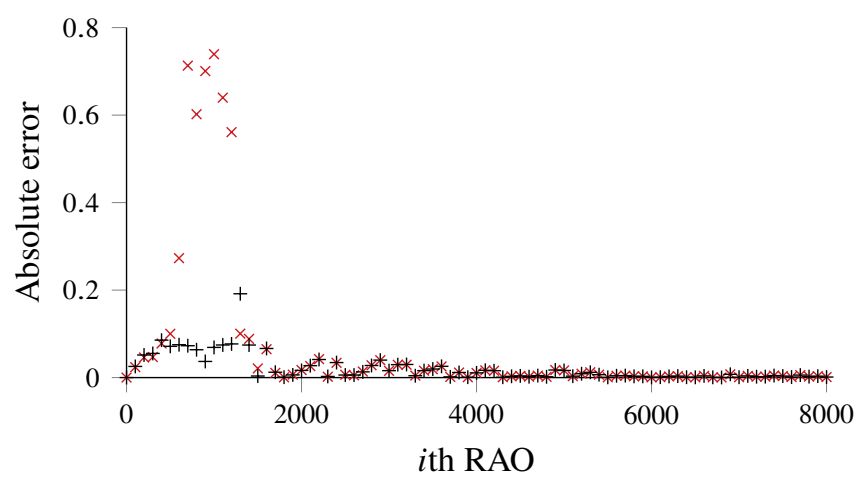

(b)

Figure 11. (a) Comparison and (b) absolute error of the expected number of successful accesses at each $\mathrm{RAO}, \mathbb{E}\left[M_{\mathrm{S}}(i)\right]$, obtained by simulation, by the extended reference model and by our model; a static ACB is implemented with $p_{\text {acb }}=0.5$ and $t_{\text {acb }}=4$.

subframes. It can be observed that the CDF of access delay presents a rapid increase, i.e., from 0 to around $p_{\text {acb }}=0.5$ (which is very close to the selected 50th percentile) in the first subframes. Then, it remains almost constant until around $d=2800$, when the UEs that succeed in their second barring check begin the RA procedure. In other words, $p_{\text {acb }}=0.5$ of the total UEs succeed in the first barring check and transmit
Table III

KPIS OBTAINED BY SIMULATION AND THE RELATIVE ERROR OBTAINED BY THE EXTENDED REFERENCE MODEL (ERM) AND BY OUR PROPOSED MODEL (PM) FOR THE SELECTED SCENARIO; STATIC ACB WITH

$$
p_{\mathrm{ACB}}=0.5 \text { AND } t_{\mathrm{ACB}}=4 \text {. }
$$

\begin{tabular}{lrrrr}
\hline & & & \multicolumn{2}{c}{ Rel. error (\%) } \\
\cline { 3 - 5 } & \multicolumn{1}{c}{ KPI } & Simulation & ERM & PM \\
\hline Success probability & & $97.48 \%$ & 1.39 & 0.18 \\
$\begin{array}{l}\text { Collision probability } \\
\text { Number of preamble transmissions }\end{array}$ & $1.62 \%$ & 18.00 & 3.28 \\
& Expected value & 2.45 & 7.29 & 1.35 \\
10th percentile & 1.00 & 0.00 & 0.00 \\
& 50th percentile & 1.40 & 5.77 & 2.04 \\
90th percentile & 4.54 & 14.31 & 1.70 \\
& 95th percentile & 6.13 & 13.30 & 1.40 \\
Access delay & & & \\
& Expected value & $4141.86 \mathrm{~ms}$ & 2.36 & 2.37 \\
10th percentile & $18.12 \mathrm{~ms}$ & 4.83 & 12.32 \\
& 50th percentile & $2945.89 \mathrm{~ms}$ & 92.63 & 4.47 \\
90th percentile & $11839.26 \mathrm{~ms}$ & 1.04 & 1.04 \\
95th percentile & $15809.89 \mathrm{~ms}$ & 0.88 & 0.87 \\
\hline
\end{tabular}

their preamble immediately; the other $1-p_{\text {acb }}=0.5$ of the total UEs must wait at least $d=2800$ to transmit their preambles.

The other contributing factor is the lack of accuracy of the extended reference model in those RAOs where $\mathbb{E}\left[M_{\mathrm{S}}(i)\right] \approx$ 15 , which increases the success probability of the UEs with low delay, i.e., those that do not delay the beginning of the RA procedure due to $\mathrm{ACB}$. As a result, the CDF of access delay obtained by the extended reference model rises slightly more rapidly than the one obtained by simulations and by our model. This slight difference at the beginning of the CDF of the access delay causes a large error in the 50th percentile.

It can be seen in Table III that each and every KPI that was obtained by means of our model is extremely close to the one obtained by means of simulations. The only exception is the 10th percentile of access delay, because (for the sake of simplicity) we assume that the RV of the barring time and the RV of the backoff time are independent RVs. While in this scenario this is not the case, assuming otherwise would 


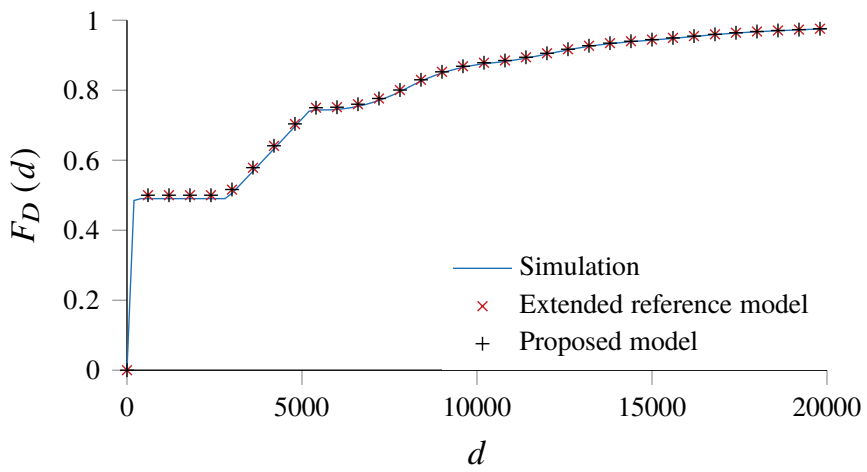

(a)

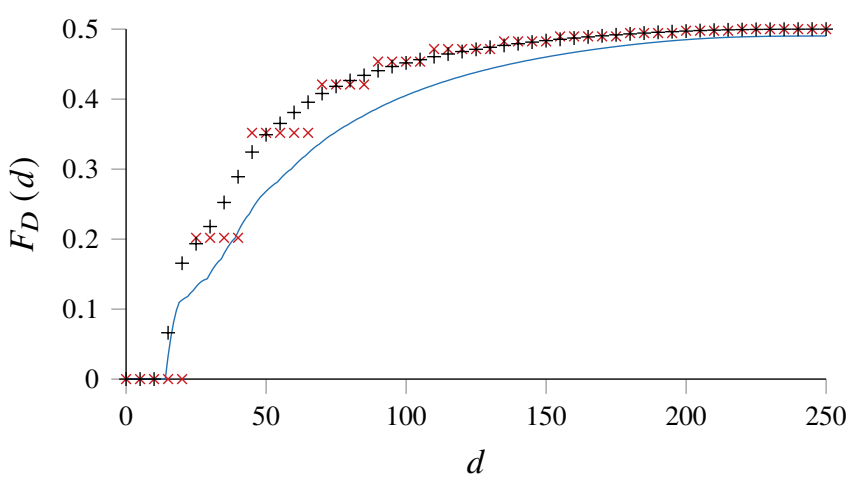

(b)

Figure 12. (a) Overall view and (b) first 250 subframes of the CDF of the access delay, $F_{D}(d)$, obtained by simulation, by the extended reference model and by our model; a static ACB is implemented with $p_{\mathrm{acb}}=0.5$ and $t_{\mathrm{acb}}=4$.

significantly increase the complexity (and computational cost) of the model.

By looking at Fig. 11a, we can observe that the UEs that succeed in the first barring check (the UEs that perform their first preamble transmission within the first 2000 RAOs) attempt to access the eNB in RAOs with a large number of contending UEs. On the other hand, the UEs that delay their preamble transmissions due to ACB attempt to access the eNB in RAOs with a low number of contending UEs. As a result, the collision probability and the backoff time is greater for those UEs that succeed in their first barring check than for those who delay their preamble transmissions. Consequently, there is a slight difference between the CDF of access delay obtained by simulation and the ones obtained by both analytic models. As Fig. 12b shows, the difference between the CDFs is noticeable in the first few subframes.

\section{The eNB decodes the preambles transmitted by multiple UEs}

As briefly explained in Section II-B, two outcomes are possible when multiple UEs transmit the same preamble at the same RAO. In the first one, the eNB does not decode these preambles; throughout this study, we have assumed that this outcome occurs every time multiple UEs transmit the same preamble. In the second one, the eNB correctly decodes these preambles. The two major effects of decoding the preambles
Table IV

KPIS OBTAINED BY SIMULATION AND THE RELATIVE ERROR OBTAINED BY THE EXTENDED REFERENCE MODEL (ERM) AND BY OUR PROPOSED MODEL (PM) FOR THE SELECTED SCENARIO; THE ENB DECODES THE PREAMBLES TRANSMITTED BY MULTIPLE UES.

\begin{tabular}{|c|c|c|c|}
\hline \multirow[b]{2}{*}{ KPI } & \multirow[b]{2}{*}{ Simulation } & \multicolumn{2}{|c|}{ Rel. error (\%) } \\
\hline & & ERM & PM \\
\hline Success probability & $16.42 \%$ & 1.36 & 0.30 \\
\hline Collision probability & $49.46 \%$ & 0.38 & 0.09 \\
\hline \multicolumn{4}{|l|}{ Number of preamble transmissions } \\
\hline Expected value & 3.43 & 1.42 & 1.48 \\
\hline 10th percentile & 1.00 & 0.00 & 0.00 \\
\hline 50th percentile & 1.88 & 2.35 & 2.66 \\
\hline 90th percentile & 7.53 & 0.66 & 0.65 \\
\hline 95 th percentile & 8.73 & 0.28 & 0.28 \\
\hline \multicolumn{4}{|l|}{ Access delay } \\
\hline Expected value & $103.38 \mathrm{~ms}$ & 3.57 & 8.27 \\
\hline 10th percentile & $15.00 \mathrm{~ms}$ & 26.67 & 3.95 \\
\hline 50th percentile & $69.83 \mathrm{~ms}$ & 24.34 & 19.23 \\
\hline 90th percentile & $256.60 \mathrm{~ms}$ & 0.44 & 5.97 \\
\hline 95th percentile & $306.21 \mathrm{~ms}$ & 5.39 & 5.33 \\
\hline
\end{tabular}

transmitted by multiple UEs are: (i) uplink grants may be sent in response to preambles transmitted by multiple UEs and (ii) the multiple UEs that receive an uplink grant will send their $\mathrm{Msg} 3 \mathrm{~s}$ in the same reserved uplink resources. As described in [35], [36], [37] these two effects negatively impact the performance of the RA procedure; their implications are described next.

If uplink grants are sent in response to preambles transmitted by multiple UEs, less than $n_{\text {ug }}$ uplink grants will be available for preambles transmitted by exactly one UE. In other words, downlink resources are wasted on UEs that have no possibility of successfully completing the RA procedure. On the other hand, if multiple UEs send their Msg3 in the same reserved uplink resources, a collision will occur at this point. Moreover, the UEs will not be aware of the collision until $h_{\text {max }} M s g 3$ s are transmitted and no Msg4 is received. That is, these UEs will be aware that a collision has occurred only after the maximum number of $M s g 3$ transmissions is reached; only then, these UEs will perform backoff. As a consequence, the delay of these UEs will increase.

We have adapted our model in order to evaluate the performance of the LTE-A RA procedure when uplink grants may be transmitted in response to preambles transmitted by multiple UEs. Table IV shows the results obtained by simulation, by adapting the reference model and by our model. The same principles employed to adapt our model were used to adapt the reference model. As it can be seen, our model exceeds the accuracy of the extended reference model in success probability, collision probability and several delay percentiles. The accuracy of both models is similar for the different metrics of the number of preamble transmissions. We have also observed that the accuracy of both models is similar when the ACB scheme is implemented; these results are not included due to the lack of space.

\section{CONCLUSION}

In this paper, we have presented a novel analytic model for the performance evaluation of the RA in LTE-A, which in- 
cludes the model of the static ACB scheme. We have assessed the accuracy of our model under several channel configurations with respect to simulation results and then compared it with that of the reference model (proposed by C. H. Wei et al.). Although the latter was the most accurate model prior to ours, its accuracy drops when the number of successful accesses per RAO approximates the system capacity, i.e., when most of the resources are being utilized. These are the scenarios of highest interest because the main objective of access control schemes is that of reducing congestion while efficiently using the available resources.

We have observed that the accuracy of our model surpasses that of the reference model. As such, our model is, to the best of our knowledge, the most accurate analytic model for the performance evaluation of the RA in LTE-A and its accuracy is not affected by the distribution of the UE arrivals; still, it maintains an acceptable degree of (computational) complexity. For instance, by implementing our model in Octave, results were obtained within a few tens of seconds for the case in which no ACB is implemented and within a couple of minutes for the case in which the static ACB is implemented.

In addition, we have adapted our model in order to evaluate the performance of the LTE-A RA procedure under the assumption that the eNB correctly decodes the preambles transmitted by multiple UEs. The process for adapting our model was also used to adapt the reference model in order to consider this assumption. Results show that the accuracy of our model is preserved.

Yet another contribution of this paper is the analytic model of the static ACB scheme. Results indicate that our model is very accurate and can be easily incorporated into other analytic models; in this case, the reference model. The only considerations that must be taken into account are (i) the percentiles of access delay that are close to the selected barring rate may be highly affected by the lack of accuracy of the selected model of the RA procedure, and (ii) in the first few subframes, the CDF of access delay obtained by our model may raise more rapidly than the one obtained by simulation.

Future work includes a refinement of the model for the ACB scheme that eliminates the error observed in the first few subframes of the CDF of access delay.

\section{REFERENCES}

[1] Architecture enhancements to facilitate communications with packet data networks and applications, 3GPP TS 23.682, Mar 2016.

[2] A. Lo, Y. Law, and M. Jacobsson, "A cellular-centric service architecture for machine-to-machine (M2M) communications," IEEE Wireless Commun. Mag., vol. 20, no. 5, pp. 143-151, 2013.

[3] M.-Y. Cheng, G.-Y. Lin, H.-Y. Wei, and A. C.-C. Hsu, "Overload control for machine-type-communications in LTE-advanced system," IEEE Commun. Mag., vol. 50, no. 6, pp. 38-45, 2012.

[4] A. Biral, M. Centenaro, A. Zanella, L. Vangelista, and M. Zorzi, "The challenges of M2M massive access in wireless cellular networks," Digit. Commun. Netw., vol. 1, no. 1, pp. 1-19, 2015.

[5] Y. Mehmood, C. Görg, M. Muehleisen, and A. Timm-Giel, "Mobile M2M communication architectures, upcoming challenges, applications, and future directions," EURASIP J. Wirel. Commun. Netw., vol. 2015, no. 1, pp. 1-37, 2015.

[6] T. Taleb and A. Kunz, "Machine type communications in 3GPP networks: potential, challenges, and solutions," IEEE Commun. Mag., vol. 50, no. 3, pp. 178-184, 2012.
[7] F. Ghavimi and H.-H. Chen, "M2M Communications in 3GPP LTE/LTEA Networks: Architectures, Service Requirements, Challenges, and Applications," IEEE Commun. Surveys Tuts., vol. 17, no. 2, pp. 525-549, 2015.

[8] P. K. Verma, R. Verma, A. Prakash, A. Agrawal, K. Naik, R. Tripathi, T. Khalifa, M. Alsabaan, T. Abdelkader, and A. Abogharaf, "Machine-toMachine (M2M) Communications: A Survey," J. Netw. Comput. Appl., vol. 66, pp. 83-105, 2016.

[9] L. Ferdouse, A. Anpalagan, and S. Misra, "Congestion and overload control techniques in massive M2M systems: a survey," Trans. Emerg. Telecommun. Technol., 2015.

[10] A. Laya, L. Alonso, and J. Alonso-Zarate, "Is the Random Access Channel of LTE and LTE-A Suitable for M2M Communications? A Survey of Alternatives," IEEE Commun. Surveys Tuts., vol. 16, no. 1, pp. 4-16, 2014.

[11] Cisco. (2016, Feb) Cisco visual networking index: Global mobile data traffic forecast update, 2015-2020. [Online] Available: http://www.cisco.com/c/en/us/solutions/collateral/serviceprovider/visual-networking-index-vni/mobile-white-paper-c11520862.html

[12] T. M. Lin, C. H. Lee, J. P. Cheng, and W. T. Chen, "PRADA: Prioritized random access with dynamic access barring for MTC in 3GPP LTE-A networks," IEEE Trans. Veh. Technol., vol. 63, no. 5, pp. 2467-2472, 2014.

[13] T. P. C. de Andrade, C. A. Astudillo, and N. L. S. da Fonseca, "Random access mechanism for RAN overload control in LTE/LTE-A networks," in Proc. IEEE International Conference Communications (ICC), 2015, pp. $5979-5984$

[14] M. Tavana, V. Shah-Mansouri, and V. W. S. Wong, "Congestion control for bursty M2M traffic in LTE networks," in Proc. IEEE International Conference on Communications (ICC), 2015, pp. 5815-5820.

[15] Z. Zhang, H. Chao, W. Wang, and X. Li, "Performance Analysis and UE-Side Improvement of Extended Access Barring for Machine Type Communications in LTE," in Proc. IEEE Vehicular Technology Conference (VTC Spring), 2014, pp. 1-5.

[16] Ping Zhou, Honglin Hu, Haifeng Wang, and Hsiao-hwa Chen, "An efficient random access scheme for OFDMA systems with implicit message transmission," IEEE Trans. Wireless Commun., vol. 7, no. 7, pp. 2790-2797, 2008.

[17] C. H. Wei, G. Bianchi, and R. G. Cheng, "Modeling and analysis of random access channels with bursty arrivals in OFDMA wireless networks," IEEE Trans. Wireless Commun., vol. 14, no. 4, pp. 1940$1953,2015$.

[18] R. G. Cheng, J. Chen, D. W. Chen, and C. H. Wei, "Modeling and analysis of an extended access barring algorithm for machine-type communications in LTE-A Networks," IEEE Trans. Wireless Commun., vol. 14, no. 6, pp. 2956-2968, 2015.

[19] O. Arouk and A. Ksentini, "General Model for RACH Procedure Performance Analysis," IEEE Commun. Lett., vol. 20, no. 2, pp. 372 $375,2016$.

[20] Service requirements for Machine-Type Communications, 3GPP TS 22.368 V13.2.0, Dec 2016.

[21] O. Galinina, A. Turlikov, T. Tirronen, J. Torsner, S. Andreev, and Y. Koucheryavy, "Random-access latency optimization and stability of highly-populated LTE-based M2M deployments," in Proc. IEEE International Conference on Communications (ICC), 2016, pp. 1-6.

[22] C. H. Wei, R. G. Cheng, and S. L. Tsao, "Performance analysis of group paging for machine-type communications in LTE networks," IEEE Trans. Veh. Technol., vol. 62, no. 7, pp. 3371-3382, 2013.

[23] O. Arouk, A. Ksentini, and T. Taleb, "How accurate is the RACH procedure model in LTE and LTE-A?" in Proc. IEEE International Wireless Communications and Mobile Computing Conference (IWCMC), 2016, pp. 61-66.

[24] M. Condoluci, G. Araniti, T. Mahmoodi, and M. Dohler, "Enabling the IoT Machine Age With 5G: Machine-Type Multicast Services for Innovative Real-Time Applications," IEEE Access, vol. 4, no. c, pp. $5555-5569,2016$

[25] I. Leyva-Mayorga, L. Tello-Oquendo, V. Pla, J. Martinez-Bauset, and V. Casares-Giner, "Performance analysis of access class barring for handling massive M2M traffic in LTE-A networks," in Proc. IEEE International Conference on Communications (ICC), 2016, pp. 1-6.

[26] Radio Resource Control (RRC); Protocol specification, 3GPP TS 36.331 V13.0.0, Jan 2016.

[27] Suyang Duan, V. Shah-Mansouri, and V. W. S. Wong, "Dynamic access class barring for M2M communications in LTE networks," in Proc. IEEE Global Telecommunications Conference (GLOBECOM), 2013, pp. 4747-4752. 
[28] Service accessibility, 3GPP TS 22.011 V13.6.0, Jul 2016.

[29] "Study on RAN Improvements for Machine-type Communications," 3GPP, TR 37.868, Jul 2011.

[30] Physical channels and modulation, 3GPP TS 36.211 V12.6.0, Sept 2015.

[31] "Feasibility study for Further Advancements for E-UTRA," 3GPP, TR 36.912 V13.0.0, Jan 2016.

[32] L. Segura, "Access control for M2M devices," Aug. 18 2011, US Patent App. 13/028,093.

[33] Medium Access Control (MAC) protocol specification, 3GPP TS 36.321 V13.0.0, Feb 2016.

[34] Physical layer procedures, 3GPP TS 36.213 V13.0.0, May 2016.

[35] T. P. C. D. Andrade, C. A. Astudillo, L. R. Sekijima, and N. L. S da Fonseca, "The Random Access Procedure in Long Term Evolution Networks for the Internet of Things," IEEE Commun. Mag., vol. 55, no. 3, pp. 124-131, 2017

[36] P. Osti, P. Lassila, S. Aalto, A. Larmo, and T. Tirronen, "Analysis of PDCCH Performance for M2M Traffic in LTE," IEEE Trans. Veh. Technol., vol. 63, no. 9, pp. 4357-4371, 2014.

[37] L. Tello-Oquendo, I. Leyva-Mayorga, V. Pla, J. Martinez-Bauset, J. R. Vidal, V. Casares-Giner, and L. Guijarro, "Performance Analysis and Optimal Access Class Barring Parameter Configuration in LTE-A Networks with Massive M2M Traffic," IEEE Trans. Veh. Technol., submitted for publication.

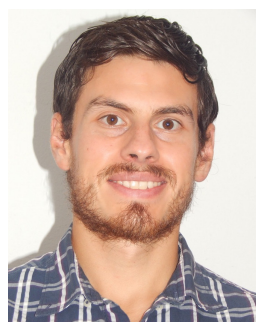

Israel Leyva-Mayorga received the B.Sc. degree in telematics engineering in 2012 and the M.Sc. degree in mobile computing systems with honorable mention in 2014, both from the Instituto Politécnico Nacional (IPN) in Mexico City, Mexico. Since 2015, he has been a Ph.D. student in telecommunications at the Communications Department of the Universitat Politècnica de València, Valencia, Spain, where he was a visiting researcher in 2014. His research interests include wireless sensor networks, communication systems, random access protocols, M2M communications, along with 5G and LTE/LTE-A networks.

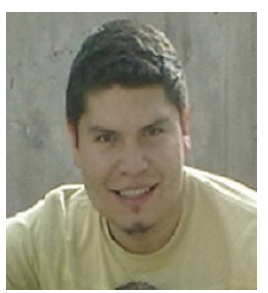

Luis Tello-Oquendo (S'08-GS'14) received the Eng. degree with 1st class honors from Escuela Superior Politécnica de Chimborazo (ESPOCH), Ecuador, in 2010, and the M.Sc. degree in Telecommunication Technologies, Systems, and Networks from Universitat Politècnica de València (UPV), Spain, in 2013. During 2011, he was a Lecturer at the Facultad de Ingeniería Electrónica, ESPOCH, Ecuador. From 2016 to 2017 he was a Visiting Research Scholar at the Broadband Wireless Networking Lab., Georgia Institute of Technology, Atlanta, GA, USA. He is currently a Graduate Research Assistant at the Broadband Internetworking Research Group, UPV, Spain. He is working toward the $\mathrm{Ph} . \mathrm{D}$. degree in Telecommunications Engineering. His research interest covers various areas of mobile and wireless communication networks, including management of massive access for machine-to-machine communications, wireless software-defined networks, LTE-A and beyond cellular systems, 5G Internet of Things. He is a member of the IEEE and ACM. He received the Best Academic Record Award from Escuela Técnica Superior de Ingenieros de Telecomunicación, UPV, in 2013, and the IEEE ComSoc Award for attending the IEEE ComSoc Summer School at The University of New Mexico, Albuquerque, NM, USA, in 2017.

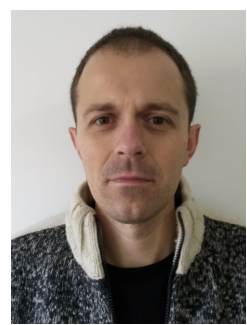

Vicent Pla received the M.E. and Ph.D. degrees in telecommunications from the Universitat Politècnica de València (UPV), Spain, and the B.Sc in Mathematics from the Universidad Nacional de Educación a Distancia, Spain. In 1999, he joined the Department of Communications, UPV, where he is currently an Associate Professor. His research interests lie primarily in the area of modeling and performance analysis of communication networks. During the past few years, most of his research activity has focused on resource management in wireless networks. In these areas he has authored numerous papers in refereed journals and conference proceedings, and has been an active participant in several national and European research projects.

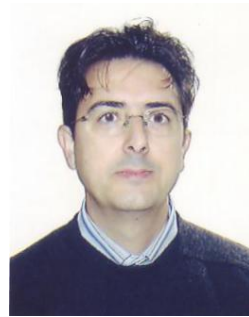

Jorge Martinez-Bauset received the Ph.D. degree from the Universitat Politècnica de València (UPV), Valencia, Spain, in 1997. He also received the 1997 Alcatel Spain Best Ph.D. Thesis Award in Access Networks. He is currently a Professor with the UPV. From 1987-1991, he was with QPSX Communications, Perth, Australia, working with the team that designed the first IEEE 802.6 MAN. Since 1991, he has been with the Department of Communications, UPV. His research interests are in the area of performance evaluation and traffic control for multiservice

networks.

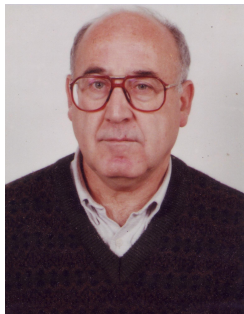

Vicente Casares-Giner (M'75-LM'17) assistant professor (1974), associate professor (1985), and full professor (1991). He obtained the Telecommunication Engineering degree in October 1974 from Escuela Técnica Superior de Ingenieros de Telecomunicación-Universidad Politécnica de Madrid (ETSIT-UPM) and the Ph.D. in Telecommunication Engineering in September 1980 from ETSIT-Universitat Politècnica de Catalunya (ETSITUPC), Barcelona. During the period 1974-1983 he worked on problems related to signal processing, image restoration, and propagation aspects of radio-link systems. In the first half of 1984 he was a visiting scholar at the KTH Royal Institute of Technology in Stockholm, dealing with digital switching and concurrent programming for Stored Program Control (SPC) telephone systems. From September 1, 1994 until August 31, 1995, he was a visiting scholar at WINLAB-Rutgers University-USA, working with random access protocols in wireless networks, wireless resource management, and land mobile trunking system. During the 90's he worked in traffic and mobility models in several European Union (EU) projects. Since September 1996, he is at ETSIT-Universitat Politècnica de València (ETSIT-UPV), Valencia, Spain. During the 00's and 10 's he has been involved in several National and EU projects. Professor V. Casares-Giner has published several papers in international magazines and conferences, such as, IEEE, Electronic Letters, Signal Processing, EURASIPEUSIPCO, International Teletraffic Conference (ITC), Wireless conferences, IEEE (ICASSP, ICC, ICUPC, ICNC,...). He has served as General co-chair in the ISCC 2005, in the NGI-2006 and as TPC member in several conferences and workshops (Networking 2011, GLOBECOM 2013, ICC 2015, VTC $2016, \ldots)$. His main interest is in the area of performance evaluation of wireless systems, in particular random access protocols, system capacity and dimensioning, mobility management, cognitive radio and wireless sensor networks. 